\title{
GATA2 is required for lymphatic vessel valve development and maintenance
}

\author{
Jan Kazenwadel, ${ }^{1}$ Kelly L. Betterman, ${ }^{1}$ Chan-Eng Chong, ${ }^{2}$ Philippa H. Stokes, ${ }^{3}$ Young K. Lee, ${ }^{2}$ Genevieve A. Secker, ${ }^{1}$ Yan Agalarov, ${ }^{4}$ \\ Cansaran Saygili Demir, ${ }^{4}$ David M. Lawrence, ${ }^{5,6}$ Drew L. Sutton, ${ }^{1}$ Sebastien P. Tabruyn, ${ }^{1}$ Naoyuki Miura, ${ }^{7}$ Marjo Salminen, ${ }^{8}$ \\ Tatiana V. Petrova, ${ }^{4,9}$ Jacqueline M. Matthews, ${ }^{3}$ Christopher N. Hahn, ${ }^{2,10}$ Hamish S. Scott, ${ }^{2,5,6,10}$ and Natasha L. Harvey ${ }^{1,10}$ \\ 'Centre for Cancer Biology, University of South Australia and SA Pathology, Adelaide, South Australia, Australia. ${ }^{2}$ Centre for Cancer Biology, University of South Australia and \\ Department of Molecular Pathology, SA Pathology, Adelaide, South Australia, Australia. ${ }^{3}$ School of Molecular Bioscience, University of Sydney, Sydney, New South Wales, Australia. \\ ${ }^{4}$ Department of Oncology, University Hospital of Lausanne and Department of Biochemistry, University of Lausanne, Lausanne, Switzerland. \\ ${ }^{5}$ Australian Cancer Research Foundation (ACRF) Cancer Cenomics Facility, Centre for Cancer Biology, University of South Australia and SA Pathology, Adelaide, South Australia, Australia. \\ ${ }^{6}$ School of Molecular and Biomedical Bioscience, University of Adelaide, Adelaide, South Australia, Australia. ${ }^{7}$ Department of Biochemistry, Hamamatsu University School of Medicine, Hamamatsu, Japan. \\ ${ }^{8}$ Department of Veterinary Biosciences, University of Helsinki, Helsinki, Finland. ${ }^{9}$ École Polytechnique Fédérale de Lausanne (EPFL), Swiss Institute for Experimental Cancer Research (ISREC), \\ Lausanne, Switzerland. ${ }^{10}$ School of Medicine, University of Adelaide, Adelaide, South Australia, Australia.
}

Heterozygous germline mutations in the zinc finger transcription factor GATA2 have recently been shown to underlie a range of clinical phenotypes, including Emberger syndrome, a disorder characterized by lymphedema and predisposition to myelodysplastic syndrome/acute myeloid leukemia (MDS/AML). Despite well-defined roles in hematopoiesis, the functions of GATA2 in the lymphatic vasculature and the mechanisms by which GATA2 mutations result in lymphedema have not been characterized. Here, we have provided a molecular explanation for lymphedema predisposition in a subset of patients with germline GATA2 mutations. Specifically, we demonstrated that Emberger-associated GATA2 missense mutations result in complete loss of GATA2 function, with respect to the capacity to regulate the transcription of genes that are important for lymphatic vessel valve development. We identified a putative enhancer element upstream of the key lymphatic transcriptional regulator PROX1 that is bound by GATA2, and the transcription factors FOXC2 and NFATC1. Emberger GATA2 missense mutants had a profoundly reduced capacity to bind this element. Conditional Gata2 deletion in mice revealed that CATA2 is required for both development and maintenance of lymphovenous and lymphatic vessel valves. Together, our data unveil essential roles for GATA2 in the lymphatic vasculature and explain why a select catalogue of human GATA2 mutations results in lymphedema.

\section{Introduction}

The discovery that GATA2 mutations underlie Emberger syndrome - a condition characterized by primary lymphedema and predisposition to myelodysplastic syndrome/acute myeloid leukemia (MDS/AML) (1) - revealed key roles for this zinc finger transcription factor in the lymphatic vasculature (2-4). Our initial investigations determined that GATA2 protein levels were most prominent in lymphatic vessel valves. This observation, coupled with our demonstration that GATA2 regulates genes important for lymphatic vessel valve development, prompted us to investigate whether GATA2 mutations result in lymphedema due to defective valve development and/or function. While several earlier studies implicated GATA2 in vascular development (5-8), the lack of an obvious vascular phenotype in Gata2 $^{-/-}$mice

\section{Related Commentary: p. 2924}

Authorship note: Jan Kazenwadel and Kelly L. Betterman contributed equally to this work.

Conflict of interest: The authors have declared that no conflict of interest exists. Submitted: September 4, 2014; Accepted: May 28, 2015.

Reference information: / Clin Invest. 2015;125(8):2979-2994. doi:10.1172/JCI78888. prior to their demise around E10.5 has precluded in depth analyses of Gata2 function in vasculogenesis and angiogenesis. SNPs in GATA2 have been associated with coronary artery disease (9-11), implying a role for GATA2 in arterial development, while ablation of gata2a in zebrafish affects morphogenesis of the dorsal aorta (12). Recent studies in which Gata2 was deleted in hematopoietic and endothelial cell compartments (13), or was disrupted due to mutation of an enhancer element important for endothelial Gata2 expression (14), suggested that Gata2 is important for vascular integrity and for efficient separation of the blood and lymphatic vascular networks $(13,14)$. The dependence of these phenotypes on endothelial versus hematopoietic GATA2 was not, however, dissected in these studies.

The lymphatic vasculature plays crucial roles in the return of interstitial fluid to the bloodstream, absorption of dietary lipids, and trafficking of immune cells. The formation of valves in collecting lymphatic vessels is a key event during maturation of the lymphatic vasculature and is crucial for lymph to be efficiently returned to the bloodstream; defects in lymphatic valve formation contribute to aberrant lymphatic function in lymphedema syndromes (15). Recent studies have begun to dissect the genes and cellular events important for valve morphogenesis in lymphatic vessels. 
Initiation of valve development is marked by the appearance of clusters of cells, often near vessel branchpoints, that exhibit high levels of the transcription factors PROX1, FOXC2, and GATA2 (16-18). These valve-forming cells reorient themselves with respect to the longitudinal axis of the vessel, extend into the vessel lumen, and form elongated valve leaflets composed of a bilayer of endothelial cells sandwiching an extracellular matrix core composed largely of Fibronectin-EIIIA (FN-EIIIA), laminin- $\alpha 5$, and EMILIN1 (19-21). Genes identified to be important for lymphatic vessel valve development include the transcription factors FOXC2 and NFATC1 $(15,20,22)$, the transmembrane ligand ephrinB2 (23), integrin- $\alpha 9$ and its ligands FN-EIIIA (19) and Emilin1 (21), gap junction proteins connexin37 (CX37) and connexin43 (CX43) $(16,24)$, NOTCH1 (25), SEMA3A together with receptor components NRP1 and PLEXINA1 (26, 27), angiopoietin2 (28, 29), TIE1 (30), and BMP-9 (31). Though the signals that initiate valve development are in large part enigmatic, the location of valves predominantly in regions of disturbed flow suggested that mechanical stimuli including shear stress might be important valve-initiating stimuli. Indeed, human lymphatic endothelial cells (hLECs) subjected to oscillatory shear stress (OSS) in vitro exhibit elevated levels of FOXC2 and Connexin37 (CX37), activation of NFATC1, and a change in their morphology from an elongated to cuboidal shape (20), as is observed in lymphatic endothelial cells within valve territories in vivo (20). The finding that venous valves share expression of lymphatic valve markers including PROX1, ephrinB2, and integrin- $\alpha 9-$ together with a dependence on ephrinB2 and integrin- $\alpha 9$ signalling for their development - suggests that common genetic pathways direct valve development in both lymphatic vessels and veins (32).

Another specialized population of valve endothelial cells comprises the lymphovenous valves (LVVs) $(33,34)$. These valves are located at the junction of the jugular and subclavian veins with the thoracic and right lymphatic ducts and function, together with platelets (35), to partition the lymphatic vasculature from the blood vasculature. Endothelial cells of the LVVs express PROX1, FOXC2, integrin- $\alpha 9$, and integrin- $\alpha 5(33,36)$, providing further evidence that valve development in distinct vascular compartments relies on common pathways. Unlike the valves within lymphatic vessels, however, the LVVs comprise one leaflet derived from venous endothelial cells and one derived from lymphatic endothelial cells. These valves are exquisitely sensitive to changes in Prox 1 dosage for their development (33); whereas lymphatic vessel valves form in Prox 1 heterozygous mice, LVVs do not. Little is known regarding the mechanisms by which these specialized valves develop. Some of the initiating signals may be distinct from those controlling lymphatic vessel valve development, given that the flow patterns and shear stress to which these venous cells are exposed are likely different to those encountered by lymphatic vessels.

The Gata family of transcription factors (GATA1-6) each contain 2 zinc fingers and bind a consensus WGATAR motif (in which W denotes A or T, and R denotes A or G) (37). Although important for neural $(38,39)$ and urogenital $(40)$ development, GATA2 has been most extensively studied in hematopoiesis, where it plays several key roles. GATA2 is crucial for hematopoietic stem cell development during both embryogenesis and adulthood (41-43), and in hematopoietic progenitor cells; it acts combinatorially within a transcription factor heptad that includes SCL, LYL1, LMO2, RUNX1, ERG, and FLI-1 (44). ChIP-Seq studies performed across a range of cell types demonstrate that GATA2 has distinct sites of chromatin occupancy and transcriptional targets in each cell type in which it acts, suggesting tissue-specific transcriptional interactions $(8,24,44-46)$. The zinc fingers of GATA2 serve at least 2 key roles: the C-terminal zinc finger mediates binding to WGATAR motifs $(47,48)$, whereas the N-terminal zinc finger mediates protein-protein interactions with transcriptional cofactors and stabilizes DNA binding (37). Many of the heterozygous GATA2 mutations identified in patients result in either premature truncation of the protein or impact the $\mathrm{C}$-terminal zinc finger by introducing missense alterations to this critical region of the protein. We (3) and others (49) have suggested that a complete loss of function of one GATA2 allele is the key factor that predisposes to lymphedema onset, given the clinical heterogeneity in symptoms observed in patients with GATA2 mutations. Most GATA2 missense mutations, including the prevalent T354M mutation (more than 50 to date), do not correlate with lymphedema. Our hypothesis that complete heterozygous loss of GATA2 function underlies lymphedema was complicated by the description of 3 Emberger syndrome patients with missense mutations R361L, C373R (2), and R396Q (4). This finding prompted us to investigate further the mechanisms by which GATA2 Emberger mutations impact on GATA2 function.

Here, we sought to determine the specific role of Gata2 in lymphatic vascular development, with a particular emphasis on valve development, by selectively inactivating Gata2 in the lymphatic vasculature. To investigate the mechanisms by which only a select catalogue of GATA2 mutations result in lymphedema, we assessed the effects of missense GATA2 Emberger mutations on the structure and binding to DNA of the GATA2 C-terminal zinc finger. Our work demonstrates that Gata2 plays key roles during lymphovenous and lymphatic vessel valve formation and provides molecular evidence that Gata2 "null" haploinsufficiency underlies the propensity to develop lymphedema, through regulation of genes, including Prox1 and Foxc2, that are important for lymphatic vessel development and valve development.

\section{Results}

GATA2 binds to and transactivates PROX1 regulatory elements. Our earlier work determined that siRNA-mediated GATA2 knockdown in primary embryonic mouse LECs resulted in reduced PROX1 levels (3). To investigate whether GATA2 regulation of Prox1 is mediated directly, we searched for consensus WGATAR binding sites in a $4-\mathrm{kb}$ region of the first intron of Prox 1 , approximately $4.5 \mathrm{~kb}$ downstream from the transcription start site (Prox 1 $+4.5 \mathrm{~kb}$ ), previously shown to be important for SOX18-mediated Prox1 expression (50). Five consensus WGATAR sites conserved between mouse and human were present in this region (Supplemental Figure 1A; supplemental material available online with this article; doi:10.1172/JCI78888DS1), and we showed that GATA2 is able to drive reporter gene expression from this element (Supplemental Figure 1B). We next compared the transcriptional activity of an allelic series of germline GATA2 mutants found in Emberger syndrome (R361L, C373R, and R396Q), together with those found in patients with hematological malignancies but no reported lymphedema (hereafter referred to as hematological 


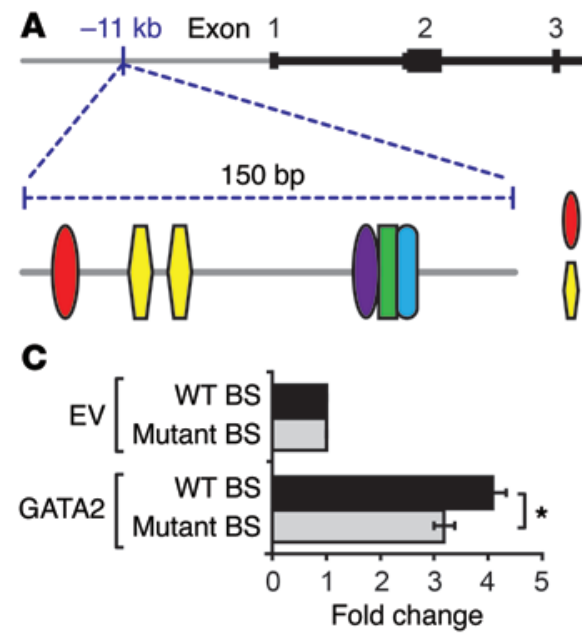

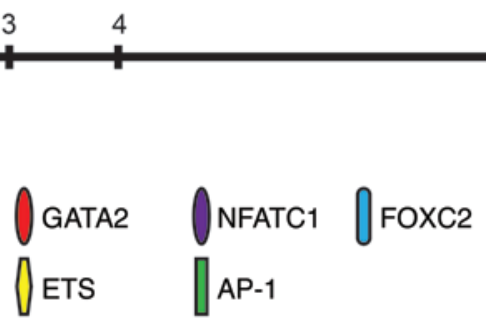

5

PROX1

D

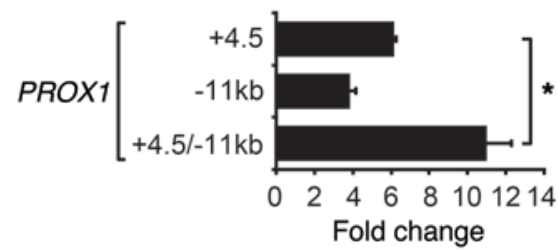

B

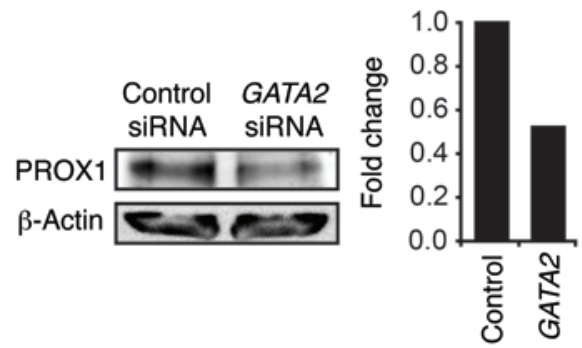

E

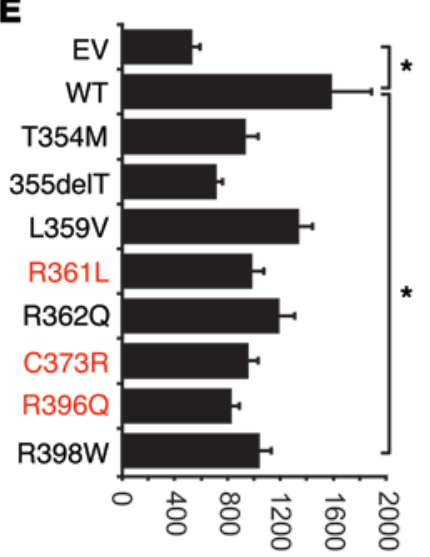

Relative intensity

$\mathbf{F}$

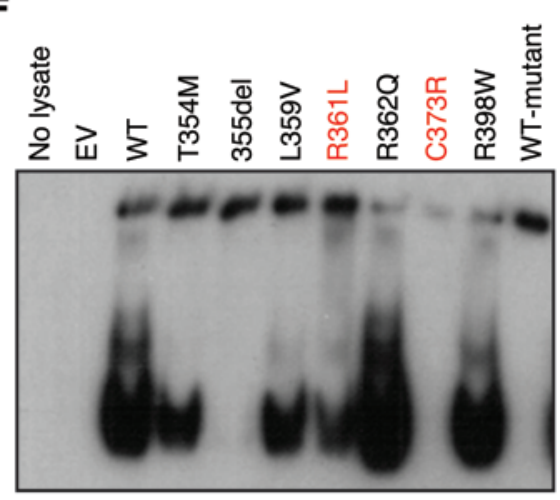

Figure 1. GATA2 Emberger mutants have reduced capacity to bind and transactivate a novel PROX1-11 kb enhancer element. (A) Schematic demonstrating location of $P R O X 1-11 \mathrm{~kb}$ enhancer element relative to the $P R O X 1$ gene and arrangement of consensus transcription factor binding sites. (B) Treatment of hLECs with GATA2 siRNA results in substantial reduction in PROX1 levels. (C) Mutation of the GATA binding site (BS) in the PROX1 -11 kb element reduced luciferase activity mediated by WT GATA2. EV, empty vector control. Error bars correspond to $\pm S E M, n=5$ independent experiments. ${ }^{*} P<0.05$, by 2-tailed Student's $t$ test. (D) The PROX1-11 kb and $+4.5 \mathrm{~kb}$ elements cooperatively enhance luciferase activity. Fold-change is shown relative to EV controls. Error bars correspond to \pm SEM, $n=3$ independent experiments. ${ }^{*} P<0.05$, by 2-tailed Student's $t$ test. (E) HEK293 cells were cotransfected with GATA2 (WT or mutant) expression constructs together with a PROX1 -11 kb luciferase reporter construct. Luciferase activity was measured after 24 hours. Error bars correspond to \pm SD $(n=6)$ from 2 independent experiments (1-way ANOVA, ${ }^{*} P<0.05$ relative to WT GATA2) Emberger mutants (here and in subsequent panels) are labeled red. (F) Binding of WT and mutant GATA2 proteins to the PROX1 -11 kb element, assessed by WEMSA.

mutants: germline T354M, 355del, R398W, somatic L359V, and R362Q), with the transcriptional activity of WT GATA2 (Supplemental Figure 1B). In all cases, the ability of GATA2 mutants to drive reporter gene expression mediated by the Prox $1+4.5 \mathrm{~kb}$ element was reduced compared with WT. The ability of GATA2 to bind to each of the 5 consensus sites in the Prox $1+4.5 \mathrm{~kb}$ element was then assessed using Western blot combined with electrophoretic mobility shift assays (WEMSA) (51). The fourth of 5 sites, located proximal to the SOX A site, displayed highest levels of binding by GATA2 (Supplemental Figure 1C), likely due to the presence of 2 overlapping GATA sites in this region. The GATA2 Emberger mutants and 355del (lacking the majority of the C-terminal zinc finger) almost completely lost the capacity to bind this site in Prox $1+4.5 \mathrm{~kb}$ (Supplemental Figure 1D). In contrast, the germline GATA2 T354M mutation - found in MDS/AML but not to date in any patients with lymphedema - and several other hematological mutants retained the ability to bind this site
(Supplemental Figure 1D). Immunoblotting of nuclear lysates was performed to ensure that comparable levels of WT and mutant GATA2 protein were present in all conditions tested in this assay (Supplemental Figure 2). Together, these data suggest that susceptibility to lymphedema might be directed by differential capacity of GATA2 mutants to bind and regulate the expression of genes important for development and function of the lymphatic vasculature, rather than hematopoiesis.

To investigate in more detail the sites bound by GATA2 in the vicinity of the PROX1 locus, we analyzed data deposited in the ENCODE database (http://genome.ucsc.edu./encode). A number of studies, including 2 undertaken in human microvascular endothelial cells (HMVECs) (24) and human umbilical vein endothelial cells (HUVECs) (8), demonstrated prominent GATA2 binding to a region $11 \mathrm{~kb}$ upstream of the first, noncoding exon of PROX1. Scanning of the DNA sequence in this peak region revealed a highly conserved region of approximately 150 nucleotides con- 


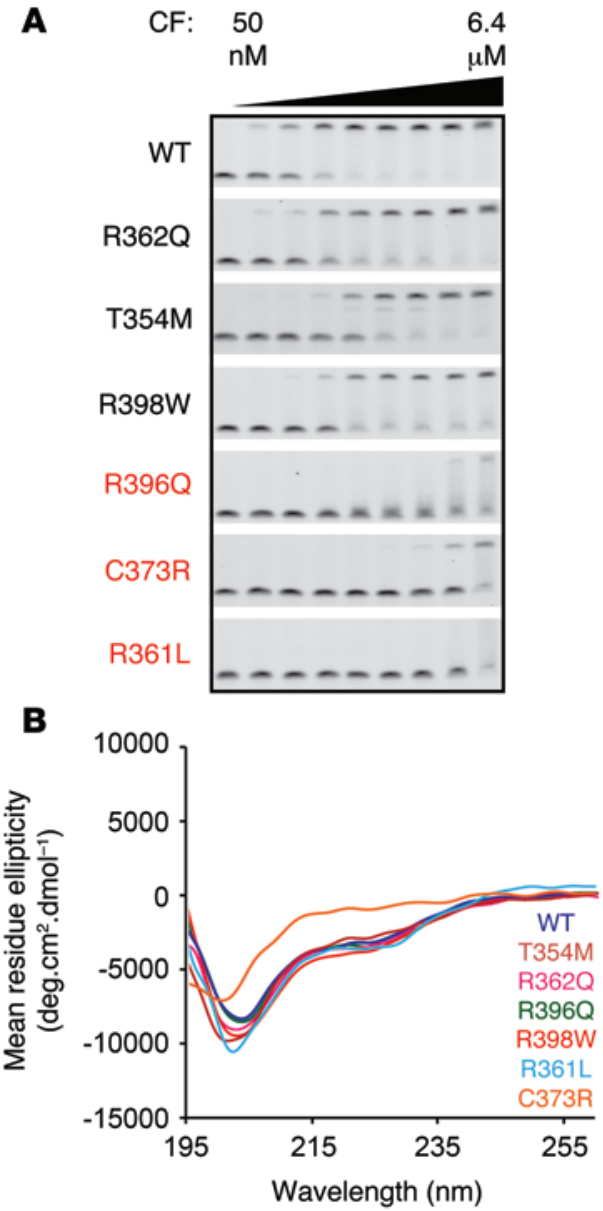

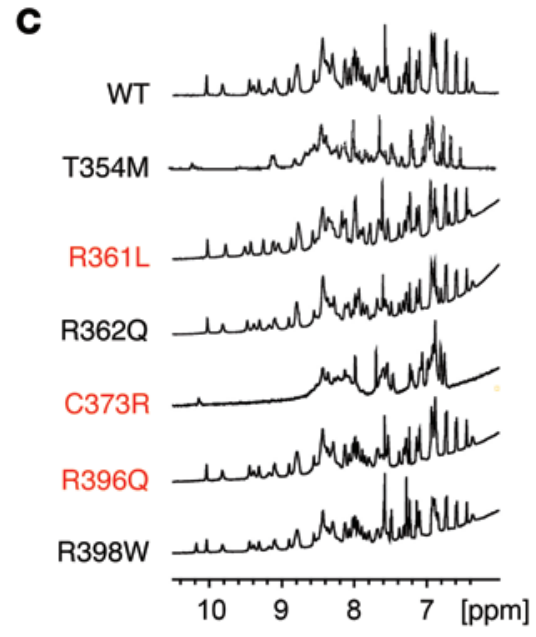

D

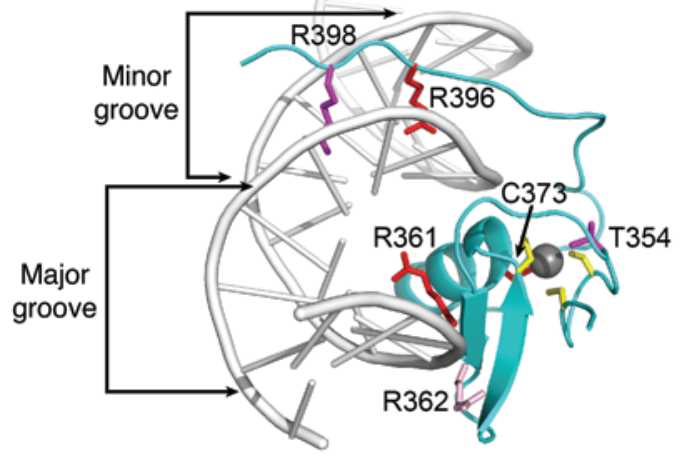

Figure 2. GATA2 Emberger mutants exhibit reduced DNA-binding affinity. (A) EMSA analyses using recombinant, purified GATA2 WT C-terminal zinc finger (CF) and mutants showing that Emberger mutants (red) exhibit negligible binding to the PROX1-11 kb element. (B) Far-UV CD spectra and (C) 1-D H1 NMR spectra (amide region) of recombinant purified proteins at $25^{\circ} \mathrm{C}, \mathrm{pH} 7.4$ in $20 \mathrm{mM}$ sodium phosphate, $50 \mathrm{mM} \mathrm{NaCl}$, $1 \mathrm{mM}$ DTT showing C373R is largely disordered and that T354M is partly disordered. (D) Structure of the C-terminal finger of GATA3 (cyan) bound to DNA (gray; Protein Data Bank accession code 4hc7 [http:// www.rcsb.org/]) showing positions of mutated residues. Emberger mutations are shown as red sticks, residues that moderately affect DNA binding by EMSA are shown as magenta sticks, and those that show WT binding are shown as pink sticks. Other zinc-coordinating residues are shown as yellow sticks, and zinc as a gray sphere. taining a WGATAR motif, together with consensus binding sites for key transcriptional regulators of lymphatic vessel valve development, FOXC2 and NFATC1 (ref. 22, Figure 1A, and Supplemental Figure 3). Moreover, hallmarks of an active enhancer element, including a DNaseI hypersensitivity site and a histone $\mathrm{H} 3$ at lysine 4 (H3K4Me1) ChIP peak, were evident in this region (see below). Treatment of adult human dermal lymphatic microvascular endothelial cells HMVEC-dLyAd (hLEC) with GATA2 siRNA confirmed that GATA2 knockdown results in substantially reduced PROX1 levels in primary adult hLECs (Figure 1B). Together, these data raised the likelihood that the $-11 \mathrm{~kb}$ region harbors an enhancer element important for regulating PROX1 expression and suggested that GATA2 binding to this element might be important for controlling PROX1 transcription.

To establish whether GATA2 binding is capable of driving reporter gene expression from the $-11 \mathrm{~kb}$ element, an $832 \mathrm{bp}$ fragment encompassing the WGATAR motif was cloned into pGL4.12 (Promega) and transfected into HEK293 cells together with an expression construct containing WT GATA2. Luciferase expression was substantially elevated in GATA2 transfected cells compared with cells transfected with a vector control (Figure 1C). Mutation of the GATA site from GATA to CTTA reduced luciferase expression by approximately $25 \%$ (Figure 1C), demonstrating that GATA2 binding to this site is important for optimal enhancer activity. The $-11 \mathrm{~kb}$ element was also cloned distally into the enhancer site of pGL4.12, together with a subcloned region of the Prox $1+4.5 \mathrm{~kb}$ ele- ment (containing GATA sites 4 and 5, see Methods for details) acting as a promoter. This addition resulted in a significant increase in luciferase activity, demonstrating that the Prox $1-11 \mathrm{~kb}$ element is capable of acting as an enhancer (Figure 1D). We next investigated the capacity of the GATA2 mutants to drive reporter gene expression from the $-11 \mathrm{~kb}$ enhancer element. All mutants demonstrated substantially reduced ability to drive reporter gene expression (Figure 1E). The relative affinity of WT and mutant GATA2 binding to the GATA site in PROX1 -11 kb was then assessed using WEMSA (51) (Figure 1F). As observed with the Prox 1 +4.5 kb GATA site (Supplemental Figure 1D), the binding of Emberger syndrome mutants R361L and C373R to the PROX1-11 kb GATA site was substantially reduced, especially in the case of C373R, where binding was completely abolished (Figure $1 \mathrm{~F}$ ). In contrast, apart from the 355del mutant, the hematological GATA2 mutants retained demonstrable binding to the PROX1-11 kb GATA site.

GATA2 Emberger mutants exhibit reduced DNA-binding affinity. The binding of GATA 2 mutants to the PROX1-11 kb GATA site was next investigated using EMSA and recombinant purified C-terminal zinc finger constructs (Figure 2A). EMSAs demonstrated that the hematological GATA2 mutant R362Q does not significantly affect binding to DNA, whereas the hematological GATA2 mutants T354M and R398W bound with moderately reduced affinity (Figure 2A). More strikingly, all 3 Emberger mutants R396Q, C373R, and R361L exhibited much lower levels of binding to DNA, with gel-shifts evident only at very high concentrations of GATA2 (Fig- 


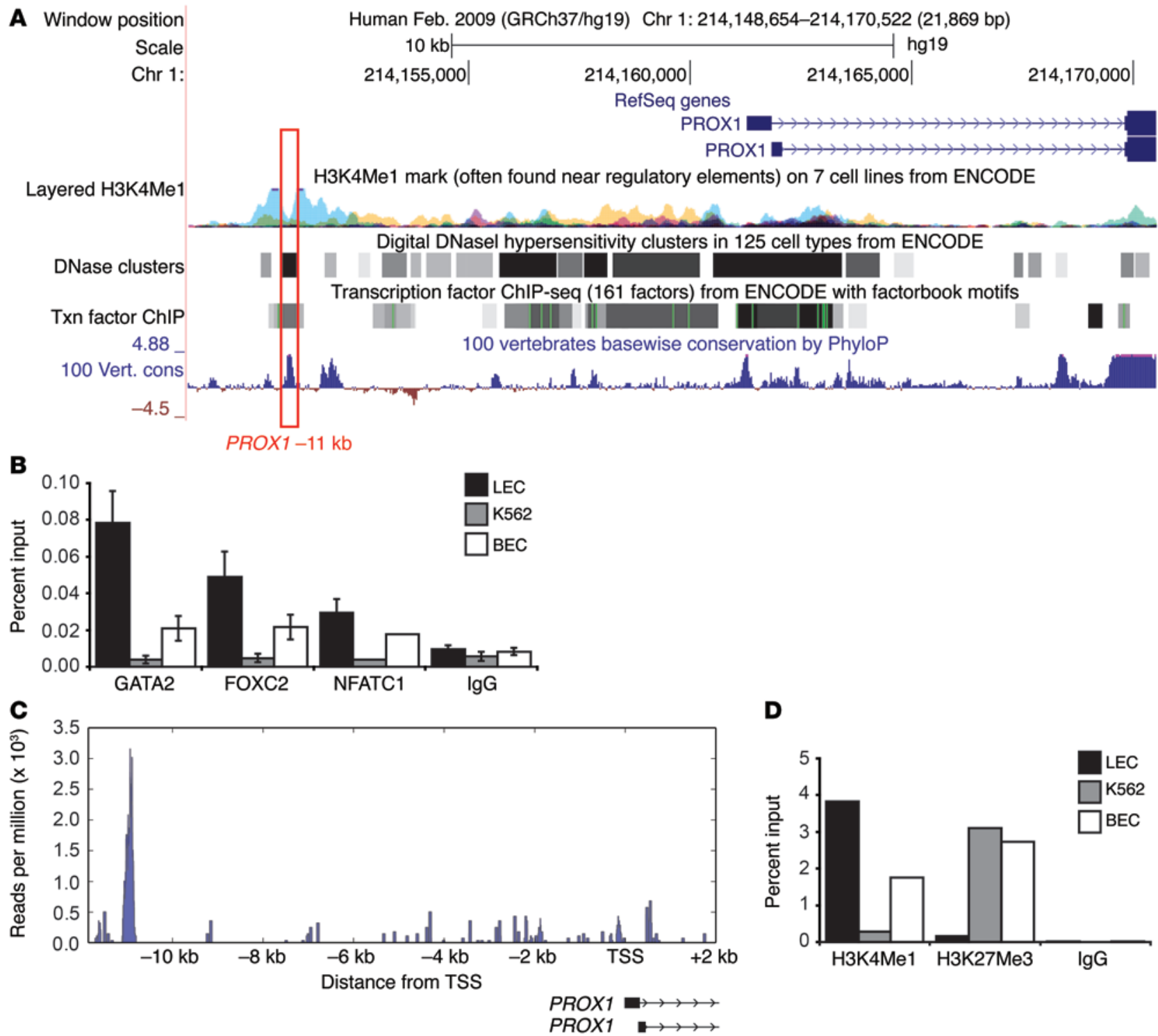

Figure 3. Occupancy of chromatin at the PROX1 -11 kb enhancer element. (A) PROX1 locus as viewed in UCSC Human Genome Browser (http://genome. ucsc.edu/). Red boxed area indicates approximate location of the PROX1-11 kb enhancer element. (B) ChIP demonstrates that GATA2, FOXC2, and NFATC1 ChIP at the PROX1-11 kb enhancer in LECs and BECs, but not K562 cells. Where error bars are shown, error bars represent \pm SEM, $n \geq 3$ independent experiments. Where error bars are not shown, data represents an average of 2 independent experiments. (C) ChIP-seq profile illustrating occupancy of the PROX1 locus by GATA2 in lymphatic endothelial cells. (D) ChIP with markers of active (H3K4Me1) and inactive/repressed (H3K27Me3) chromatin at the PROX1 -11 kb element demonstrates that $P R O X 1$-11 kb is active in LECs and repressed in BECs and K562. Data are representative of 2 independent experiments.

ure 2A). The purity of GATA2 WT and Emberger zinc fingers was assessed by SDS-PAGE analysis prior to use in EMSA assays (Supplemental Figure 4). Folding of these mutants was assessed by farUV circular dichroism (CD) spectroscopy and 1D $\mathrm{H}^{1}$-NMR spectroscopy (Figure 2, B and C) to assess secondary-structure content and overall fold, respectively. The CD data indicates that all mutants apart from C373R have WT-like levels of secondary structure. Both the CD and NMR spectra for C373R are characteristic of a largely disordered protein domain with a blue-shifted minimum in the CD spectrum and poor peak dispersion in the NMR spectrum. T354M also shows some disruptions to structure in the NMR spectrum with peak dispersion intermediate between that of the WT and C373R proteins. These data indicate that mutation of a zinc-coordinating residue, C373, prevents the GATA2 C-ter- minal zinc finger from folding and binding to DNA. The T354M mutation appears to be molten globule-like with high levels of secondary but poorly packed tertiary structure. This smaller disruption to folding allows the protein to bind DNA, probably through a binding and folding mechanism, albeit with reduced affinity. The structure of the GATA2 C-terminal zinc finger has not been determined. However, the zinc finger domains of GATA1-3 are highly conserved, with all mutated residues being identical (Supplemental Figure 5). A homology model of the GATA2 C-terminal zinc finger (Supplemental Figure 5), based on GATA3 bound to DNA (52) indicates that the Emberger R361L and R396Q mutations likely disrupt binding to DNA by mutation of key residues that interact with the major and minor grooves, respectively (Figure 2D). R362 makes some minor interactions with phosphates on the backbone 


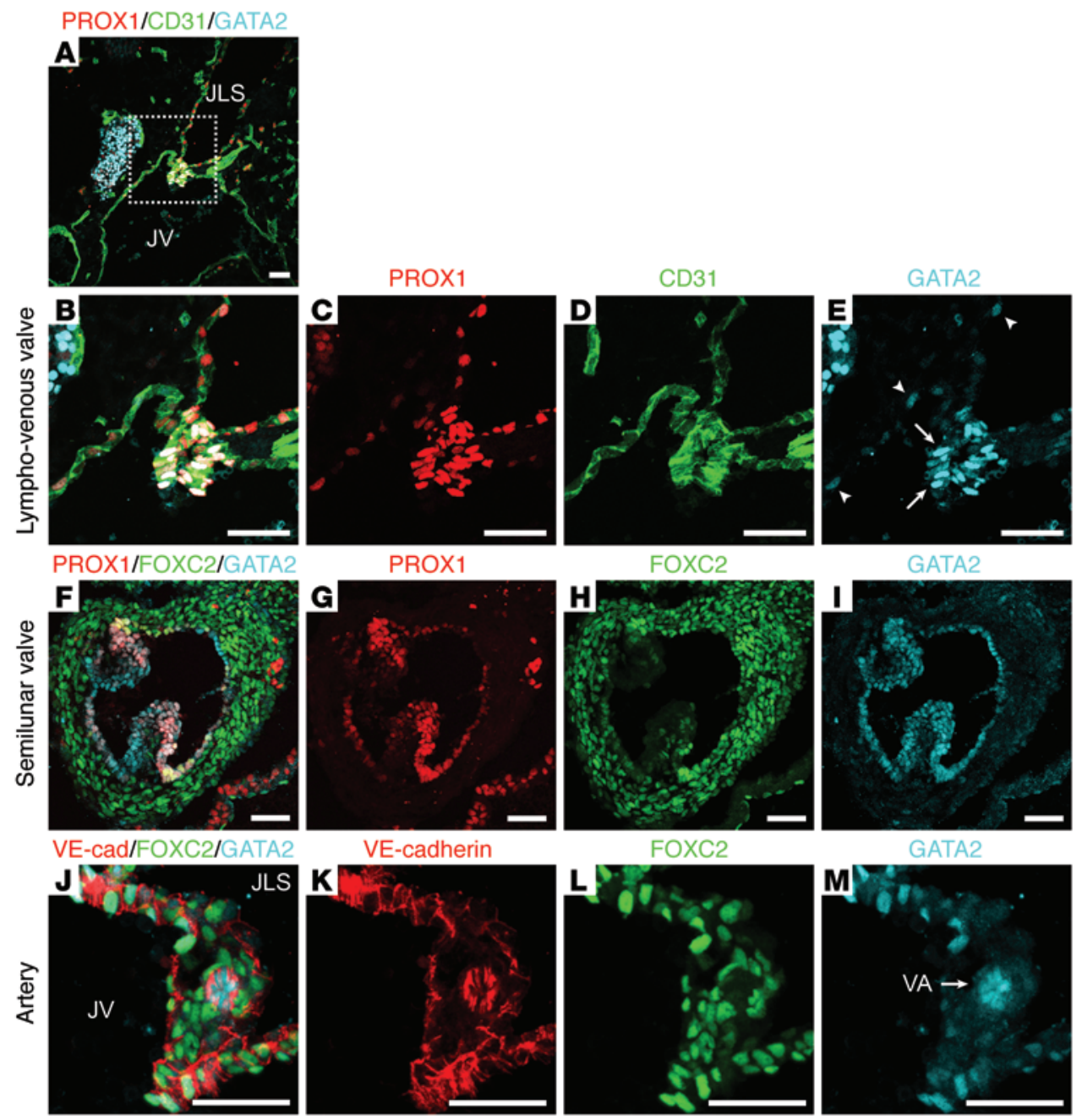

Figure 4. Localization of GATA2 in valves and arteries. (A-E) Immunostaining of E13.5 WT mouse tissue sections demonstrated that GATA 2 is present at higher levels in the endothelial cells comprising LVV leaflets (E, arrows) compared with endothelial cells lining the jugular veins and jugular lymph sacs (E, arrowheads). GATA2 is also present in cardiac valves $(\mathbf{F}-\mathbf{I})$ and arterial endothelial cells (J-M). Boxed region in $\mathbf{A}$ is shown at a higher magnification in B-E. Scale bars: $50 \mu \mathrm{m}$. JLS, jugular lymph sac; JV, jugular vein; VA, vertebral artery. of DNA, but this interaction is fairly solvent exposed, and mutation to polar glutamine likely has very little effect on DNA binding. Based on data for GATA3-DNA interactions, R398 should not play a direct role in binding to WGATAR sites (52) (but is involved in binding to pseudo-palindromic CTACTGATA sites through binding in the minor groove), so moderate loss of DNA binding likely arises from loss of long-range electrostatic interactions between the positively charged arginine sidechain and the negatively charged DNA. Overall, these data support the hypothesis that substantial losses in PROX1 -11 kb binding, through mutation of key structural or DNA-interacting residues in the C-terminal zinc finger of GATA2, correlate with lymphedema.

The PROX1 -11 kb locus is differentially regulated in lymphatic compared with blood vascular endothelial cells. We next investigated the binding of GATA2 to the PROX1-11 kb region in both adult human dermal lymphatic microvascular endothelial cells (hLEC) and adult human dermal blood microvascular endothelial cells (hBECs) using ChIP. Hallmarks of an active enhancer element, including a DNaseI hypersensitivity site and an H3K4Me1 ChIP peak, were evident in this region (Figure 3A). Substantial occupancy of GATA2 at the $-11 \mathrm{~kb}$ site was obvious in hLECs, using both ChIP (Figure 3B) and ChIP-Seq approaches (Figure 3C). ChIP experiments also detected GATA2 binding, though to a lesser extent, at the PROX1-11 kb region in hBECs (Figure 3B). In contrast, no significant occupancy at this site was detected in erythroleukemic K562 cells (Figure 3B), which express GATA2 but not PROX1. Given our observation that consensus sites for FOX and NFAT transcription factors lie in close proximity to the GATA site in PROX1-11 kb, we next employed ChIP to investigate the occupancy of chromatin by FOXC2 and NFATC1 in hLECs, hBECs, and K562 cells. As with GATA2, marked occupancy of the PROX1-11 $\mathrm{kb}$ region by both FOXC2 and NFATC1 was observed in hLECs, and to a lesser extent hBECs, but not in K562 cells (Figure 3B). Given that FOXC2 and NFATC1 have been shown to physically associate and cooperatively regulate transcription (22), we investigated potential protein-protein interactions between GATA2, NFATC1, and FOXC2 using coimmunoprecipitation. We confirmed an interaction between FOXC2 and NFATC1 in HEK293 cells ectopically expressing these proteins, but no interaction was detected between GATA2 and FOXC2, nor between GATA2 and NFATC1 (Supplemental Figure 6).

With the exception of the embryonic cardinal veins (53), LVVs (33), and venous valves (32), substantial levels of PROX1 are not detected in blood vascular endothelial cells. We reasoned that reduced binding of GATA2, FOXC2, and NFATC1 at PROX1-11 $\mathrm{kb}$ in hBECs compared with hLECs was not sufficient to explain 

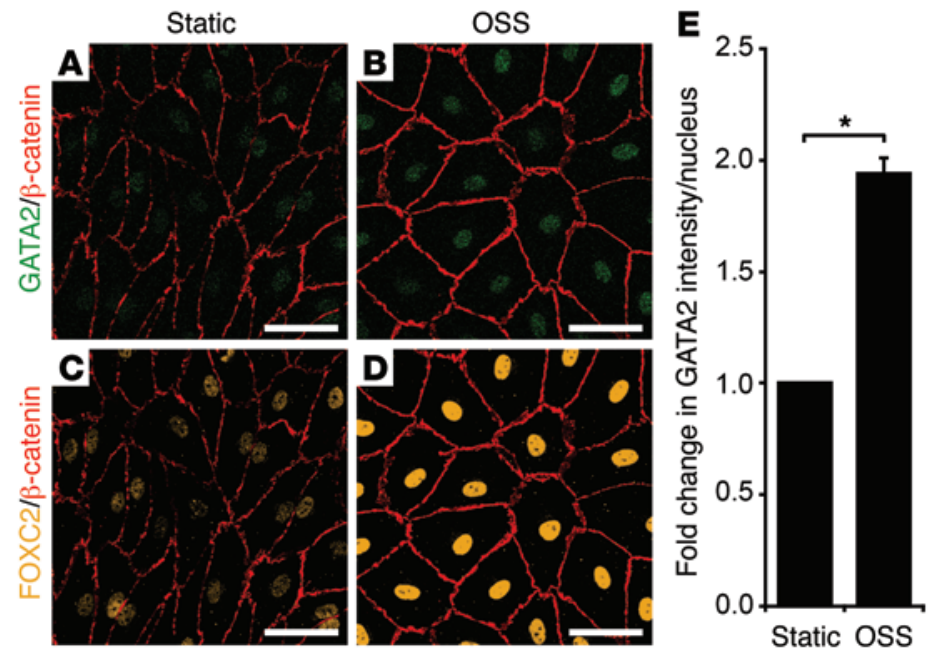

Figure 5. In vitro OSS increases GATA2 levels in hLECs. hLECs were cultured under static conditions (A and $\mathbf{C}$ ) or subjected to OSS (4 dyn $/ \mathrm{cm}^{2}$, $1 / 4 \mathrm{~Hz}$ ) (B and $\mathbf{D}$ ) for 48 hours. Immunostaining for GATA2 and FOXC2 demonstrated elevated levels of nuclear GATA2 (A, B, and E) and FOXC2 (C and D) following exposure of hLECs to OSS. Error bars correspond to \pm SEM, $n=4$ independent experiments. ${ }^{*} P<0.00001$, by 2 -tailed Student's $t$ test. Scale bars: $50 \mu \mathrm{m}$.

why $P R O X 1$ is generally silent in hBECs. To investigate the mechanisms by which the PROX1-11 kb region may contribute to turning PROX1 "on" in LECs and "off" in BECs, we investigated the status of chromatin in the PROX1-11 kb region in hLECs, hBECs, and $\mathrm{K} 562$ cells. ChIP for a marker of active chromatin, H3K4Me1, demonstrated substantial levels of this histone mark at PROX1-11 $\mathrm{kb}$ in hLECs, much lower levels in hBECs, and none in K562 cells (Figure 3D), consistent with the transcription factor analysis. Conversely, no association of the repressive histone mark histone $\mathrm{H} 3$ on lysine 27 (H3K27Me3) was detected at PROX1-11 kb in hLECs, while this mark was present in hBECs and K562 cells (Figure 3D). Together, these data provide further evidence that the PROX1-11 $\mathrm{kb}$ region harbors an enhancer element important for regulating $P R O X 1$ expression in the vasculature and suggest that chromatin modifications that act to silence this region are important in keeping PROX1 switched off in blood vessels.

GATA2 is present at prominent levels in lymphatic vessel valves, LVVs, cardiac valves, and arteries. In addition to lymphedema, other cardiovascular phenotypes described in patients harboring GATA2 mutations or polymorphisms include venous thromboses, culture negative endocarditis (49), and susceptibility to coronary artery disease. Given that our early work documented high levels of GATA2 in lymphatic vessel valves (3), we analyzed GATA2 levels in LVVs and cardiac valves. Prominent levels of GATA2 were observed in the endothelial cells that comprise LVVs (Figure 4, A-E). In comparison to the low level of GATA2 protein obvious in the endothelial cells lining the jugular vein and the lymph sacs (Figure 4E, arrowheads), GATA2 levels in the cells comprising the valve leaflets was greatly elevated (Figure 4E, arrows). GATA2 was also obvious, together with prominent PROX1 and more restricted FOXC2 staining, in semilunar valves of the embryonic heart (Figure 4, F-I). Taken together, these data suggest that - like PROX1, FOXC2, and NFATC1 - GATA2 marks valve endothelial cells across distinct vascular compartments. GATA2 protein was also obvious in arterial endothelial cells at a discernibly higher level than in veins and lymphatic vessels, though at a lower level than that present in valve endothelial cells (Figure 4, J-M).

GATA2 levels are elevated in response to oscillatory flow. To investigate the mechanisms by which GATA2 is elevated in valves, we assessed the effects of exposing hLECs to oscillatory flow in vitro. Previous work established that subjection of hLECs to OSS, reflective of the turbulent flow pattern characteristic of vessel branchpoints, promoted the acquisition of many of the cellular characteristics of valve-forming cells (16). These features include cytoskeletal remodeling and adoption of a cuboidal rather than elongated cell shape, activation of calcineurin/NFAT signaling, and elevation of CX37 levels. Moreover, acquisition of these characteristics was found to be dependent on PROX1 and FOXC2 (16). Our previous work demonstrated that reduction of Gata2 levels in primary mouse LECs (mLECs) resulted in greatly diminished levels of both Prox1 and Foxc2, suggesting that GATA2 might lie upstream of these key transcription factors in the hierarchy of events required for the initiation of valve development (3). Taking these data into account, we reasoned that GATA2 may be responsive to mechanical stimuli including shear stress and hypothesized that elevated GATA2 levels may be required for the induction of FOXC2 levels mediated by OSS. Exposure of hLECs to OSS for 48 hours resulted in elevated GATA2 mRNA levels and significantly higher levels of GATA2 protein in the nuclei of hLECs (Figure 5, A, B, and E, and Figure 6, A and C). Accordingly, hLECs adopted a characteristic cuboidal morphology in response to OSS, and the levels of nuclear FOXC2 were markedly elevated (Figure 5, C, D, and Figure 6, G-I). To assess whether the elevation in FOXC2 levels in response to OSS was dependent on GATA2, hLECs were treated with control (Figure 6, A-C, and G-I) or GATA2 siRNA (Figure 6, D-F, and J-L) and exposed to laminar (Figure 6, B, E, H, and K) or oscillatory (Figure 6, C, F, I, and L) flow. Strikingly, FOXC2 levels were much lower in GATA2 siRNA-treated cells exposed to OSS (Figure 6L) compared with control siRNA-treated cells (Figure 6I). These data suggest that OSS, typical of turbulent flow at vessel branchpoints, may be one of the stimuli by which GATA2 levels are elevated in developing valves and reveal that elevation of FOXC2 levels in response to OSS is dependent on GATA2.

GATA2 is required for lymphatic vascular development. In order to assess the requirement for GATA2 for lymphatic vascular development and, in particular, for valve development, we utilized a number of approaches to conditionally delete Gata2 in the vasculature. The first was to delete Gata2 in vascular and hematopoietic compartments by crossing Tie2-Cre mice (54) 


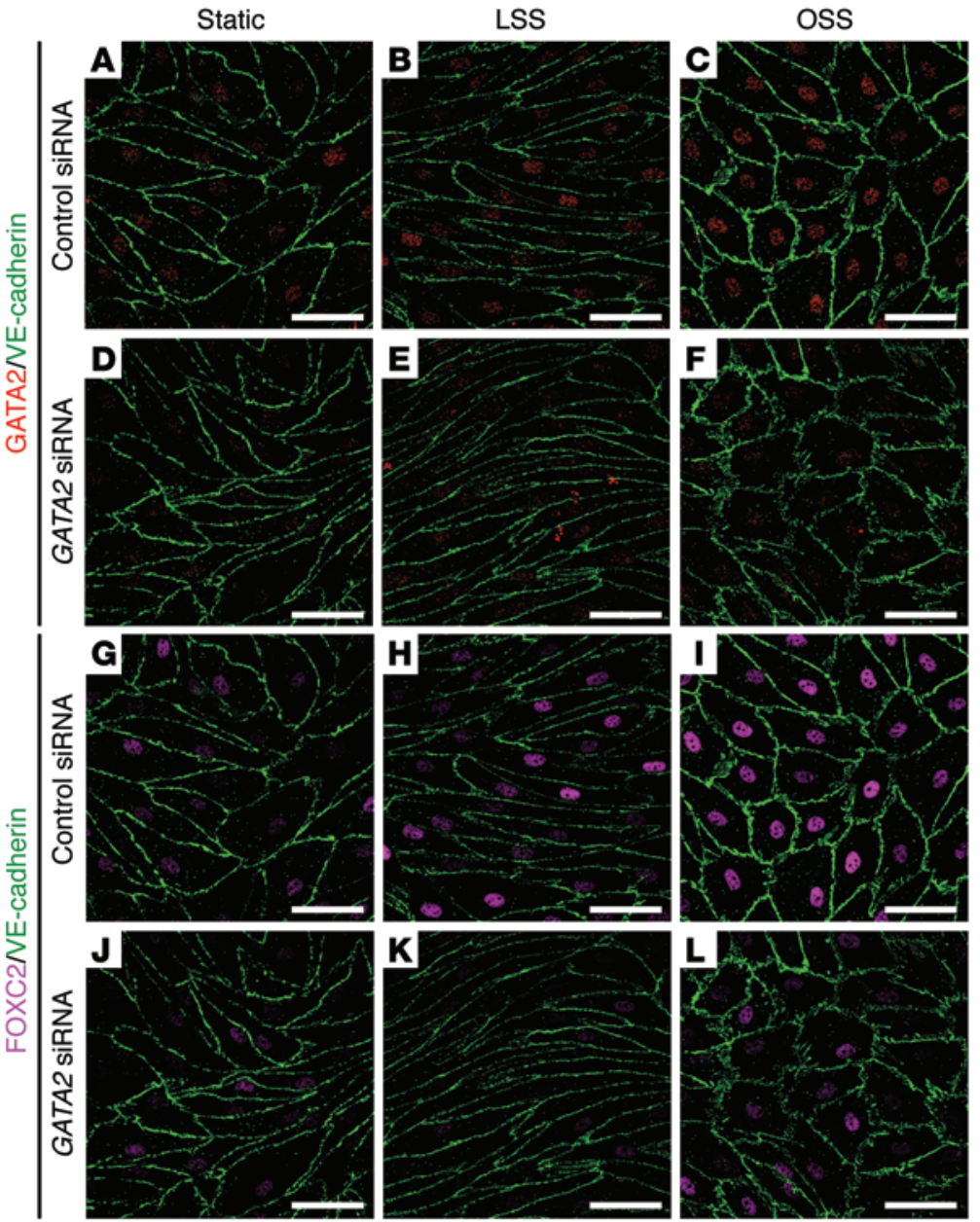

Figure 6. OSS induced elevation of FOXC2 is dependent on GATA2. hLECs were transfected with control or GATA2 siRNA and cultured under static (A, D, G, and J) conditions, or subjected to laminar shear stress (LSS; B, E, H, and $\mathbf{K}$ ) or OSS (C, F, I, and L) for 48 hours (4 dyn $/ \mathrm{cm}^{2}, 1 / 4 \mathrm{~Hz}$ ). Immunostaining revealed reduced nuclear FOXC2 levels in CATA2 siRNA-treated hLECs in response to OSS ( $\mathbf{L}$ compared with I). Scale bars: $50 \mu \mathrm{m}$. compared with I, L, and M). To investigate the underlying cause of enlarged jugular lymph sacs, we quantified proliferating lymphatic endothelial cells in the jugular lymph sacs of Gata ${ }^{\triangle E C}$ embryos and their control littermates; the number of Ki67-positive, proliferating lymphatic endothelial cells was elevated approximately 4-fold in E13.5 Gata ${ }^{\triangle E C}$ embryos (Supplemental Figure 7). To investigate the nature of the blood-filled lymphatic phenotype, we analyzed the structure of LVVs, which function together with platelet clotting to prevent blood from entering the lymphatic vasculature $(33,35)$. At E13.5, the leaflets of the LVVs appeared multilayered in Gata ${ }^{\triangle E C}$ embryos compared with their control counterparts (Figure 7, O and P, compared with $\mathrm{J}$ and $\mathrm{K}$ ), suggesting that structural abnormalities in LVV development might permit the entry of blood to the jugular lymph sacs. In addition, the levels of PROX1 observed in the venous leaflets of the LVVs (PROX1-positive, Podoplanin-negative, Endomucin-low cells) were substantially lower in Gata ${ }^{\triangle E C}$ embryos compared with their control counterparts (Figure 7, P compared with $\mathrm{K}$, arrows), suggesting that the identity and function of venous valve endothelial cells might be affected. Potential defects in platelet clotting as a result of Gata2 deletion in both hematopoietic and endothelial compartments in these mice might also contribute to the failure of effective separation of the blood and lymphatic vascular networks. Blood-filled jugular lymph sacs and dermal lymphatic vessels (Figure 7, Q and R, compared with $\mathrm{L}$ and $\mathrm{M}$ ) and focal hemorrhages (Figure $7 \mathrm{~N}$ ) were also observed in the skin of Gata ${ }^{\triangle E C}$ embryos compared with control (Figure 7I). These data reveal that Gata2 is not required for the initiation of Prox 1 expression, nor for the specification and early migration of lymphatic endothelial progenitor cells, but is required for normal LVV development and effective separation of the blood vasculature from the lymphatic vasculature.

We next investigated the consequences of selective Gata2 deletion in the lymphatic vasculature by crossing Prox1-Cre $e^{\text {ERT2 }}$ mice (56) with Gata $^{f / f l}$ mice and inducing Gata2 deletion at a range of embryonic stages (55) (Gata2 ${ }^{\triangle L E C}$ ). To compare the consequences of selectively deleting Gata2 in the lymphatic vasculature to pan-endothelial and hematopoietic deletion, we administered tamoxifen at E10.5, E11.5, and E12.5 and analyzed embryos at E13.5 (Figure 8, A-D), or administered tamoxifen at E10.5 and E11.5, analyzing embryos at E14.5 (Figure 8, E-L). Using both regimes, Gata2 ${ }^{\triangle L E C}$ embryos exhibited pronounced edema (Figure 8, C and I, arrows) and enlarged jugular lymph sacs. The phenotype of blood-filled jugular lymph sacs and dermal lymphatic vessels was, however, most prominent with the E10.5, E11.5, and E12.5 tamoxifen regime. Due to the extremely edematous nature of the embryos, it was not possible to obtain sections of sufficiently intact morphology for the analysis of LVV development, though it is presumable that the blood-filled lymphatic phenotype would likely occur as a result of defective LVV development. In the Gata2 ${ }^{\triangle L E C}$ embryos that were administered tamoxifen at E10.5 and E11.5 and analyzed at E14.5, though edema was obvious, blood- 

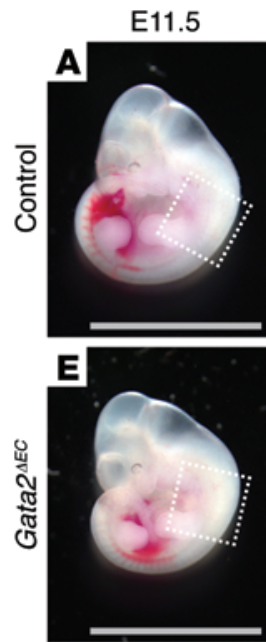

E13.5
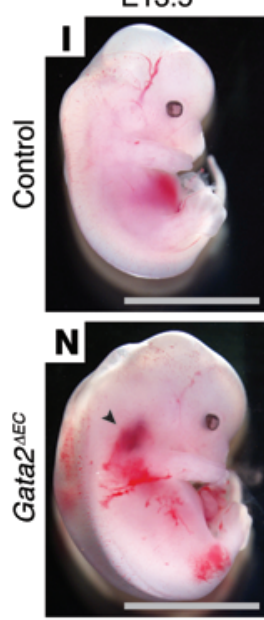

PROX1/LYVE-1
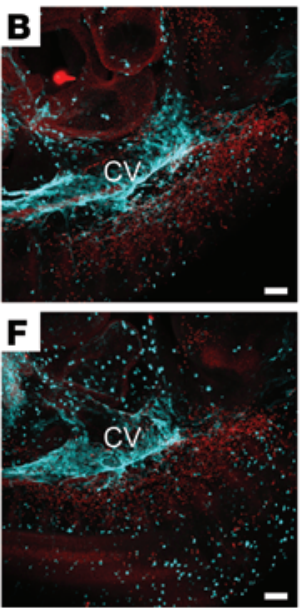

PROX1/Podo/Endd
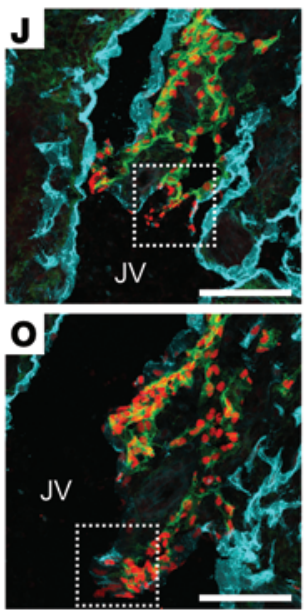

PROX1/NRP2
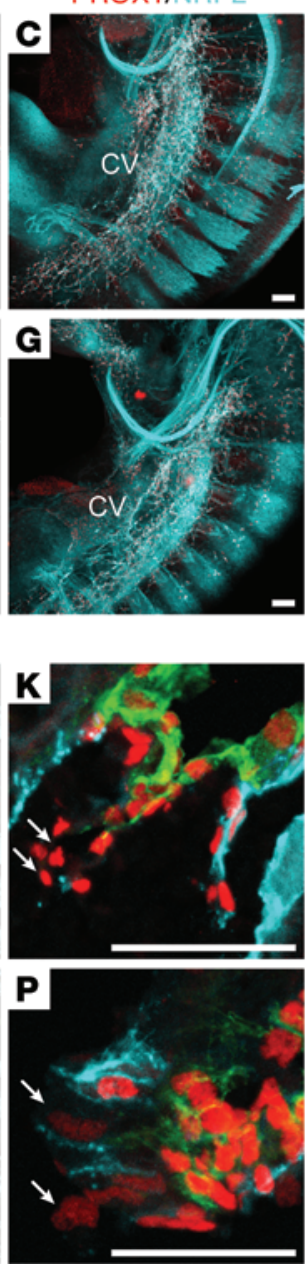

aSMA/PROX1/CD31
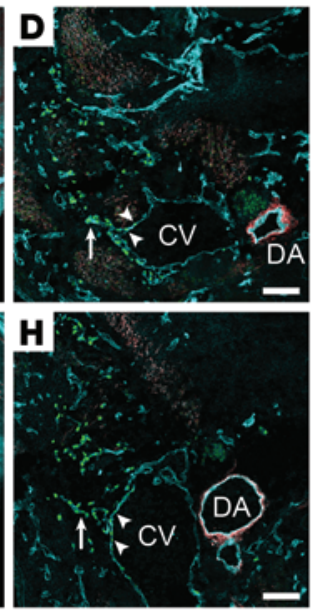

PROX1/LYVE-1/Lin-

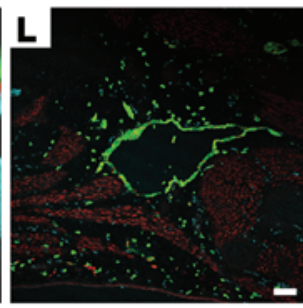

PROX1/LYVE-1/Lin+
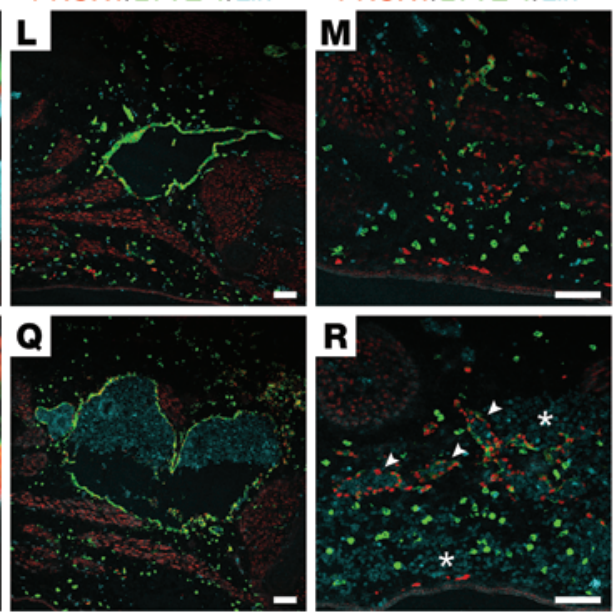

Figure 7. Lymphatic vessel hyperplasia in Gata2 ${ }^{4 E C}$ embryos. E11.5 Gata2 $2^{4 E C}$ embryos appear phenotypically normal (A and E). Whole-mount immunostaining for PROX1 (red) and LYVE-1 or NRP2 (cyan) demonstrated that PROX1-positive lymphatic endothelial progenitor cells are specified and migrate away from the cardinal veins of Gata $2^{\triangle E C}$ embryos (F and $\left.\mathbf{G}\right)$. Immunostaining of transverse sections for $\alpha$ smooth muscle actin ( $\alpha$ SMA; red), PROX1 (green), and CD31 (cyan) confirmed PROX1 expression in lymphatic endothelial progenitor cells of the cardinal veins of Gata2 ${ }^{\mathrm{AEC}}$ embryos (D and $\mathbf{H}$; arrowheads) and migration of these cells from the veins ( $\mathbf{D}$ and $\mathbf{H}$, arrows). Boxed regions in $\mathbf{A}-\mathbf{E}$ correspond to areas shown in $\mathbf{B}, \mathbf{C}, \mathbf{F}$, and $\mathbf{G}$. E13.5 Gata2 ${ }^{4 E C}$ embryos exhibit severe edema, pooling of blood in the jugular region ( $\mathbf{N}$, arrowhead), and focal dermal hemorrhages (R, asterisks), compared with control littermates

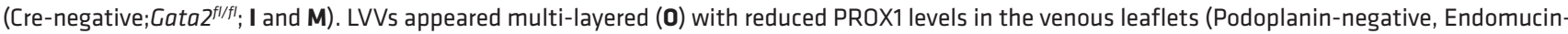
low) of Gata ${ }^{4 E C}$ embryos (P, arrows) compared with controls (J and $\left.\mathbf{K}\right)$. PROX1 levels in lymphatic leaflets (Podoplanin-positive, Endomucin-negative) of Gata ${ }^{\Lambda E C}$ embryos were not substantially altered $(\mathbf{O}$ and $\mathbf{P})$. Jugular lymph sacs $(\mathbf{Q})$ and dermal lymphatic vessels $(\mathbf{R}$, arrowheads) were enlarged and blood-filled in Gata2 ${ }^{\Delta E C}$ embryos, phenotypes not observed in control embryos ( $\mathbf{L}$ and $\mathbf{M}$ ). Magnified images of boxed regions in $\mathbf{J}$ and $\mathbf{O}$ are shown in $\mathbf{K}$ and $\mathbf{P}$, respectively. Gray scale bars: $5 \mathrm{~mm}$. White scale bars: $50 \mu \mathrm{m}$ (K and P) or $100 \mu \mathrm{m}$ (all panels excluding $\mathbf{K}$ and $\mathbf{P}$ ). CV, cardinal vein; DA, dorsal aorta; JV, jugular vein; Lin+, APC mouse lineage antibody cocktail used to detect hematopoietic cells.

filled lymphatics were not apparent, and accordingly, no striking abnormalities in LVV development were observed (Figure 8, E-L). In the majority of cases, GATA2 remained detectable in at least a few cells in the LVV region in of E14.5 embryos that had been administered tamoxifen at E10.5 and E11.5 (Figure 8K). Taken together, these data suggest that administration of tamoxifen at E12.5 could be a crucial determinant of Gata2 excision efficiency in the LVV compartment.

To investigate the consequences of Gata2 deletion on lymphatic vessel valve development, which is initiated in the dermal lymphatic vasculature at approximately E16.5, tamoxifen was administered to pregnant females at E12.5, E13.5, and E14.5, and embryos were analyzed at E16.5 and at E18.5. Analysis of embryos at E16.5 revealed that Gata $a^{\triangle L E C}$ embryos exhibited edema, bloodfilled dermal lymphatic vessels, and pooling of blood in the jugular region: phenotypes not observed in control littermates (Figure 9, A and F). Whole-mount immunostaining of embryonic skin illustrated that the dermal lymphatic vessels were bulbous, irregular in shape, and blood filled (Figure 9, B-E and G-J). In addition, ectopic association of vascular smooth muscle cells (SMCs) with dermal lymphatic vessels was observed in Gata $^{\triangle L E C}$ embryos (Figure 9H, arrows), a phenotype reminiscent of that observed in $\mathrm{Foxc2}^{-/-}$mice (15). Accordingly, FOXC2 levels appeared much lower in the lymphatic vessels of Gata ${ }^{\triangle L E C}$ embryos compared with their control counterparts (Supplemental Figure 8). Analysis of our GATA2 ChIP-Seq data revealed a prominent GATA2 bind- 


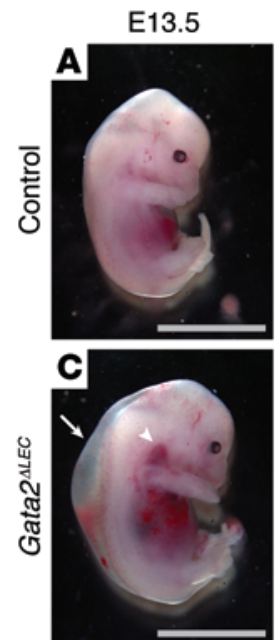

E14.5
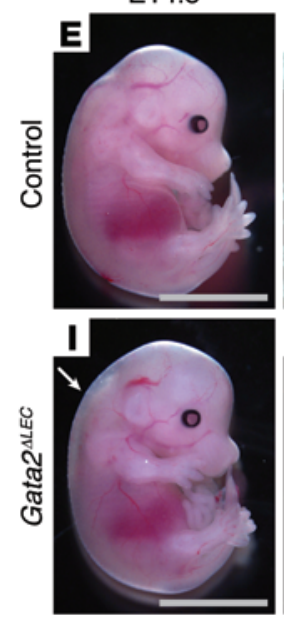
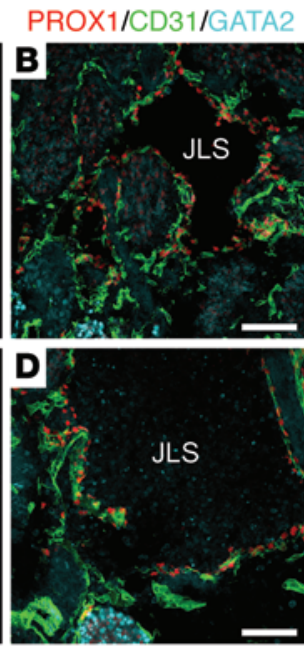

GATA2/Podo/Endo
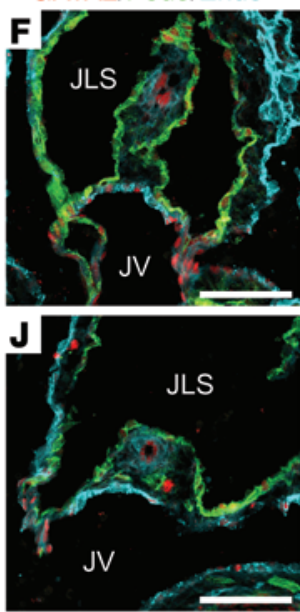

GATA2
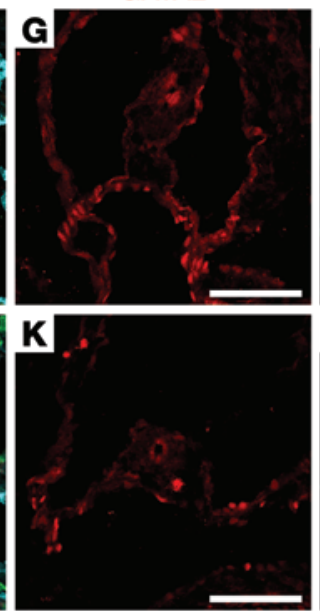
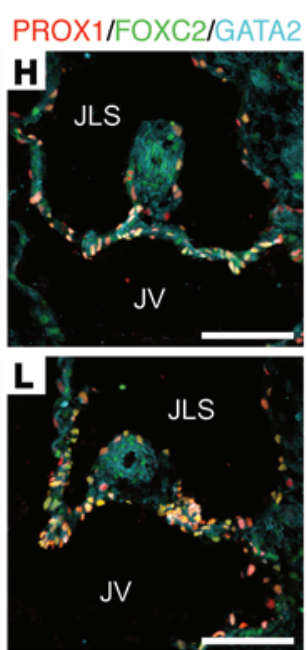

Figure 8. Lymphatic vascular phenotypes in E13.5 and E14.5 Gata2 ${ }^{\text {ALEC }}$ embryos. Prox1$\mathrm{Cre}^{\text {ERT2 }} \mathrm{Cata}^{\mathrm{fl} /+}$ male mice were crossed with Cata2 ${ }^{f / f l}$ female mice, and tamoxifen was administered at E10.5, E11.5, and E12.5, followed by analysis at E13.5 (A-D), or E10.5 and 11.5, followed by analysis at E14.5 (E-L). E13.5 Gata2 ${ }^{\perp L E C}$ embryos exhibit striking edema (C, arrow) and greatly enlarged, blood-filled jugular lymph sacs (arrowhead, C and D), compared with control littermates (Cre-negative;

Gata $f^{f / / f l}$, A and B). At E14.5, Gata2 ${ }^{\text {ALEC }}$ embryos exhibit edema (I, arrow). Immunostaining of coronal E14.5 tissue sections demonstrated no obvious morphological abnormalities in LVVs of Gata2 ${ }^{\Lambda L E C}$ mice (J-L) compared with control littermates (F-H). Scale bars: $5 \mathrm{~mm}(\mathbf{A}$ C, E, and I) or $100 \mu \mathrm{m}$ (B, D, F-H, and J-L). JLS, jugular lymph sac; JV, jugular vein. ing peak $17 \mathrm{~kb}$ downstream of the FOXC2 gene in hLECs (Supplemental Figure 9A), and the ability of GATA2 to drive reporter gene expression from this element was confirmed in luciferase assays (Supplemental Figure 9B). Together with our previous data demonstrating that Gata2 knockdown results in decreased Foxc2 levels in primary embryonic mLECs (3), these data suggest that GATA2 regulates Foxc2 expression in the lymphatic vasculature and that the $+17 \mathrm{~kb}$ element is potentially an important site for GATA2-mediated control of Foxc2.

The number of clusters of PROX1- and FOXC2-high cells at lymphatic vessel branchpoints in the skin, indicative of initial valve-forming territories, also appeared reduced in Gata ${ }^{\triangle L E C}$ embryos at E16.5 (Figure 9, D and I, arrowheads), suggesting that the initiation of valve development might be affected as a result of Gata2 deletion. To investigate a potential defect in valve development more closely, we assessed lymphatic vessel development in the mesentery of Gata ${ }^{\triangle L E C}$ embryos and their control littermate counterparts (Cre-negative;Gata $2^{f / f l}$ ) at E18.5. Striking defects in lymphatic vessel structure were observed in Gata $^{\triangle L E C}$ embryos at this stage of development; lymphatic vessels appeared bulbous and were poorly organized (Figure $9, \mathrm{~K}-\mathrm{V})$. Moreover, in contrast to control littermates in which tightly organized valve-forming territories were obvious (Figure $9, \mathrm{~L}, \mathrm{P}$, and $\mathrm{S}-\mathrm{V})$, no sign of valve development was apparent in
Gata $^{\triangle L E C}$ mesenteries. Likewise, lymphatic vessels appeared bulbous, and valve development appeared to be perturbed in the skin of E18.5 Gata ${ }^{\triangle L E C}$ embryos (Supplemental Figure 10). Taken together, our data illustrate that Gata2 is essential for lymphatic vessel valve development to be initiated.

To assess the consequences of Gata2 deletion on later stages of valve maturation, we next administered tamoxifen to pups at P4. Assessment of valve morphology and PROX1 levels in the valves of mesenteric collecting vessels at $\mathrm{P} 8$ revealed substantially reduced PROX1 levels in the valves of $\mathrm{Gata}^{\triangle L E C}$ pups (Figure 10, C, D, E, F, $\mathrm{H}, \mathrm{I}, \mathrm{K}$, and L) compared with controls (Cre-negative;Gata $2^{f / f l}$, Figure 10, A, B, G, and J). In addition, cells within the valve territories appeared more disorganized (Figure 10, C, E, H, and I). Investigation of $\beta$-galactosidase levels in the mesenteric lymphatic vessel valves of P8 Prox1-Cre ${ }^{E R T 2} \mathrm{Gata} 2^{f l /+}$ ROSA26R mice administered a single dose of tamoxifen at P4 confirmed efficient Cre activity in lymphatic vessel valves (Supplemental Figure 11, A-P). Moreover, PROX1 levels were reduced in the valves of Prox1-Cre ${ }^{E R T 2} \mathrm{Gata}^{\mathrm{fl} /+}$ ROSA26R mice (Supplemental Figure 11, G and O) and the cells in which $\beta$-galactosidase levels were highest correlated with the most obvious reduction in PROX1 levels (Supplemental Figure 11, F-H, and N-P). To investigate later-stage consequences of Gata2 deletion on the mesenteric lymphatic vasculature, pups administered tamoxifen at $\mathrm{P} 4$ were analyzed at $\mathrm{P} 10$. The phenotypes of dysmor- 

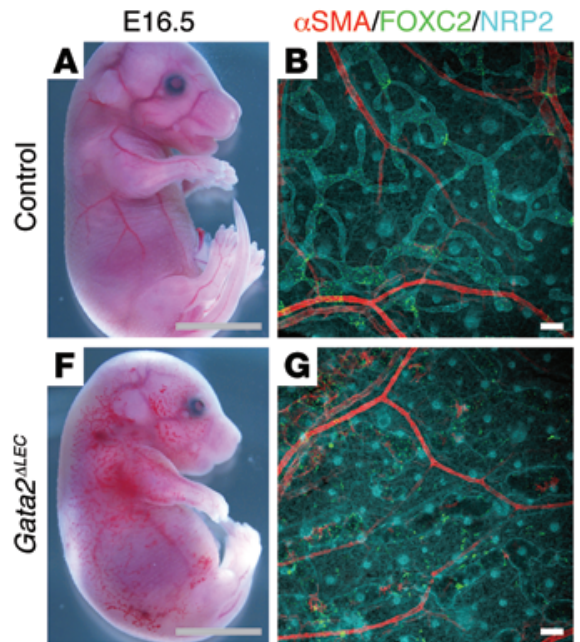

E18.5
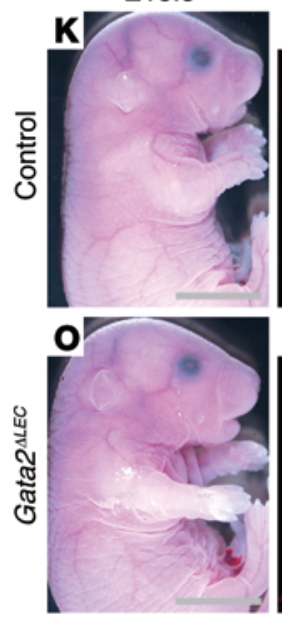
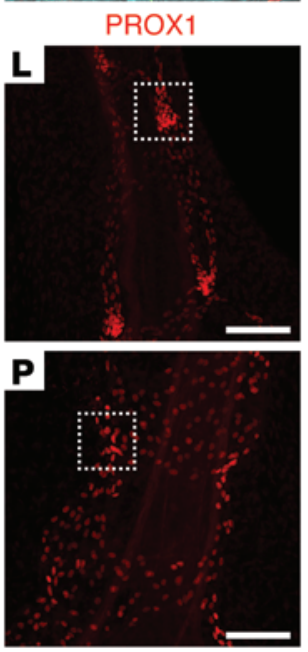
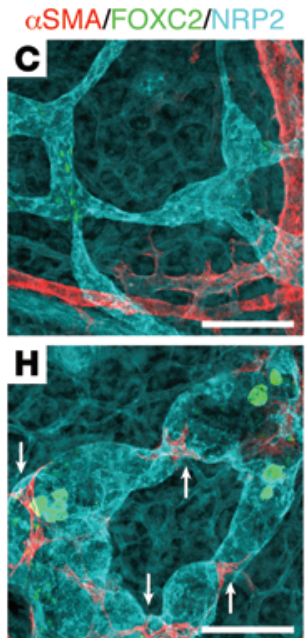

CD31
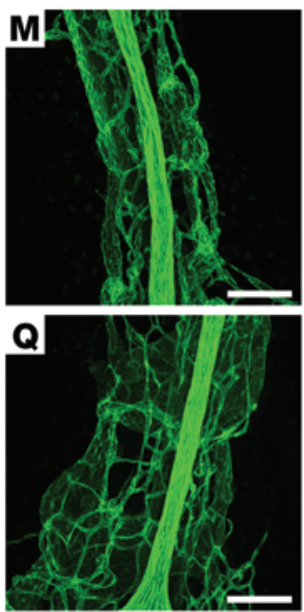

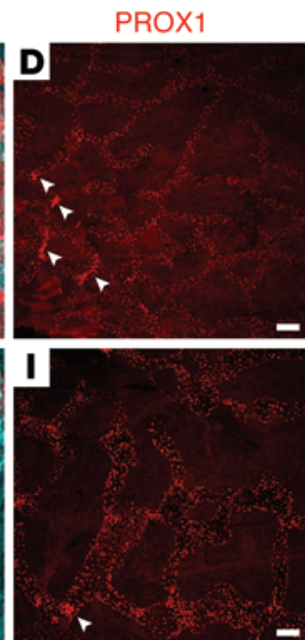

PROX1/CD31
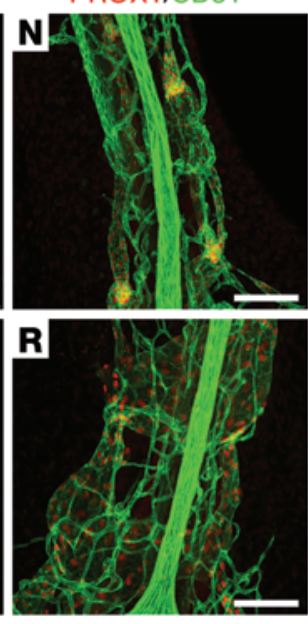

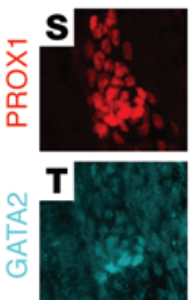

QSMA/PROX1/Lin
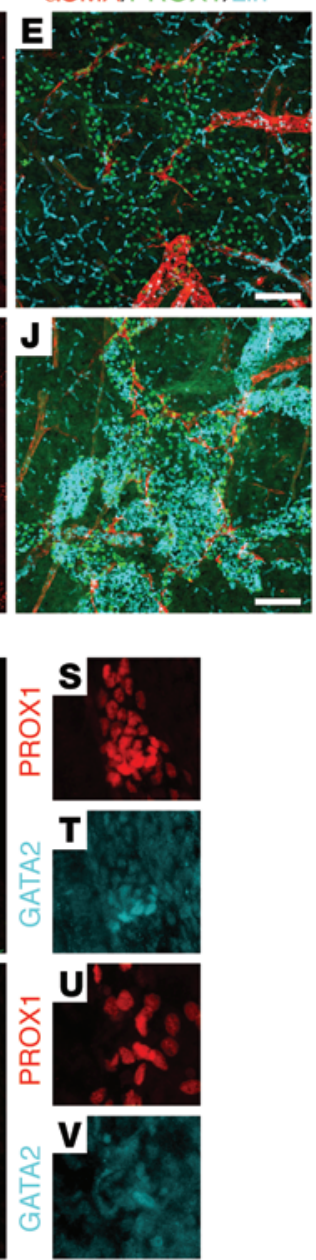

Figure 9. Lymphatic vessel valve development is perturbed in Gata2 $2^{4 L E C}$ embryos. Prox1-Cre ${ }^{E R T 2}$ Gata $2^{f /+}$ male mice were crossed with Gata $f^{f / f f}$ female mice, and tamoxifen was administered at E12.5, E13.5, and E14.5. E16.5 Gata2 ${ }^{4 L E C}$ embryos exhibit blood-filled dermal lymphatic vessels and pooling of blood in the jugular region (F), phenotypes not observed in control littermates (Cre-negative;Gata2 fl/fl, A). Whole-mount immunostaining of skin from E16.5 Gata $2^{\triangle L E C}$ embryos revealed enlarged, blood-filled dermal lymphatic vessels (G-J) that were ectopically associated with vascular SMCs (H, arrows), phenotypes not observed in control littermates (B-E). Reduced numbers of PROX1-high valve territories (I, arrowheads) were obvious in E16.5 Gata2 ${ }^{4 L E C}$ embryos compared with littermate controls (D, arrowheads). Gata2 ${ }^{\Lambda L E C}$ embryos appeared indistinguishable from control littermates at E18.5 (K and $\mathbf{0}$ ). Whole-mount immunostaining of E18.5 mesenteries demonstrated bulbous mesenteric lymphatic vessels in Cata2 ${ }^{\triangle L E C}$ embryos (P-R), lacking organized valve forming territories (L-N). Single-channel images of boxed regions in $\mathbf{L}$ and $\mathbf{P}$ are shown in $\mathbf{S}$ and $\mathbf{T}$, and $\mathbf{U}$ and $\mathbf{V}$, respectively. Scale bars: $5 \mathrm{~mm}(\mathbf{A}, \mathbf{F}, \mathbf{K}$, and $\mathbf{O})$ or $100 \mu \mathrm{m}(\mathbf{B}-\mathbf{E}$, G-J, L-N, and P-R). Lin'; APC mouse lineage antibody cocktail used to detect hematopoietic cells.

phic valves and bulbous lymphatic vessels were substantially more severe at this stage, suggesting a progressive decline in valve organization following Gata2 deletion (Figure 11). Like at P8, PROX1 levels were decreased in some valve territories of Gata $^{4 L E C}$ pups at P10, though intriguingly in other cases, PROX1 levels remained high in valves and were elevated in the lymphangion regions of mesenteric vessels. In all cases, the cells within valve territories were markedly disorganized (Figure 11, J-L, and N-P). We hypothesize that the elevation of PROX1 levels observed at P1O is a secondary effect of disrupted flow and vessel distension that occurs as a consequence of the loss of valve function.

To investigate the capacity of the lymphatic vasculature to efficiently transport lymph in the setting of Gata2 deficiency, we injected adult Gata ${ }^{A E C /+}$ mice with Evans Blue dye and monitored the transport of dye from the footpads to the thoracic duct. In the majority of cases, Evans Blue was visible in the thoracic ducts of Gata ${ }^{A E C /+}$ mice 1 hour following injection (Figure 12, A and B), though in 2 of $9 \mathrm{Gata}^{4 \mathrm{EC} /+}$ mice, Evans Blue was barely detectable. In 2 cases, blood was observed in the thoracic ducts of adult $\mathrm{Gata}^{\triangle \mathrm{EC} / \mathrm{+}}$ mice (Figure 12C). Quantification of thoracic duct areas in a cohort of mice revealed that the thoracic ducts of adult $G a t a^{\triangle E C /+}$ mice were significantly larger in calibre than their control counterparts (Figure 12, A-D). Taken together, these data illustrate alterations in lymphatic vessel structure and deficiencies in lymphatic vascular transport function in adult $\mathrm{Gata}^{\Delta E C /+}$ mice.

\section{Discussion}

Here, we identify a crucial role for Gata2 in lymphatic vascular development and, in particular, demonstrate that Gata2 is required for the formation and maintenance of lymphovenous and lymphatic vessel valves. Gata2-deficient lymphatic vessels appear bulbous, are aberrantly invested with vascular SMCs and 


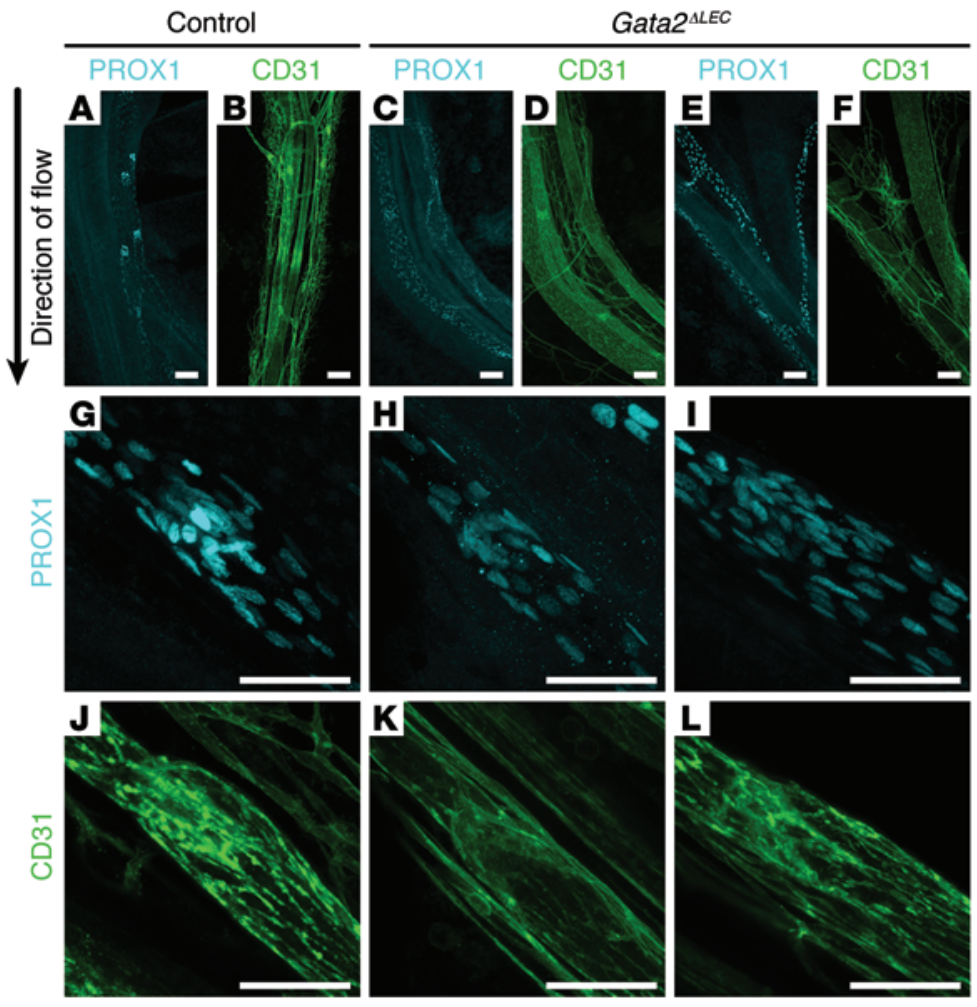

Figure 10. Gata2 is required for lymphatic vessel valve maintenance. Gata2 ${ }^{\triangle L E C}$ and littermate control (Cre-negative; Gata $\left.2^{f / / f}\right)$ pups were injected with tamoxifen at P4. Whole-mount immunostaining of mesenteries at P8 with PROX1 (cyan) and CD31 (green) demonstrated reduced PROX1 levels in lymphatic vessel valves of Gata2 ${ }^{\text {ILEC }}$ mice (C, E, H, and I), compared with littermate controls (A and $\mathbf{G})$. Blood vascular patterning was indistinguishable from littermate controls. Scale bars: $100 \mu \mathrm{m}$ (A-F) or $50 \mu \mathrm{m}$ (G-L).

exhibit dysmorphic lymphatic vessel valves (Figure 13). Underpinning this phenotype, we identify putative enhancer elements bound by GATA2 at both PROX1 and FOXC2 loci and reveal that neither of these transcription factors is elevated in prospective valve-forming territories in the absence of Gata2. Intriguingly, our data suggest that GATA2 binding at the PROX1-11 kb locus is not required for the initiation of PROX1 expression in lymphatic endothelial progenitor cells in the cardinal veins but may be important as a rheostat to "dial up" PROX1 levels in valve-forming endothelial cells. We furthermore demonstrate that GATA2 is mechanoresponsive and is elevated in hLECs in response to OSS, providing a mechanism by which GATA2 levels may be increased to initiate the process of valve development. Our data provide new insight to the mechanisms by which only a select catalogue of GATA2 mutations result in primary lymphedema by revealing that missense mutations from Emberger patients, but not those found in hematological disorders in the absence of lymphedema, result in near complete loss of GATA2 function with respect to their ability to regulate the expression of genes important for lymphatic vessel valve development, including Prox1.

Though enigmatic to date, work from multiple studies has implicated GATA2 in vascular development. An enhancer element located in the fourth intron of Gata2, also known as the +9.5 element (57), has been reported to drive reporter gene expression uniformly through the vasculature (5), though our immunostain- ing analyses revealed very low levels of GATA2 protein throughout the majority of the embryonic vasculature. Notably higher protein levels were obvious in arterial endothelial cells and, most prominently, in the cells that comprise lymphovenous and lymphatic vessel valves. One explanation for the divergence between Gata2 mRNA and protein levels could be differential posttranscriptional/ posttranslational regulation in distinct vascular beds; indeed, our earlier work documenting that miR-451 levels are elevated in primary embryonic BECs compared with LECs (3) provides one potential mechanism by which this could be accomplished. An important role for GATA2 in arteries may underlie the hemorrhagic phenotype of Gata ${ }^{\triangle E C}$ mice described by us and others, as well as the association of GATA2 SNPs in patients with early-onset coronary artery disease (9-11). Targeted mutation of gata2a in zebrafish has recently been demonstrated to result in defective morphogenesis of the dorsal aorta, resulting in arterial-venous shunts and suggesting that arterial defects could also contribute to the vascular phenotype observed in Gata ${ }^{\triangle E C}$ mice. To date, however, we have not observed any striking abnormalities in dorsal aorta morphology (12).

Conditional deletion of Gata2 throughout the vascular and hematopoietic compartments using either $+9.5 \mathrm{~kb}$ enhancer-driven (13) or VE-cadherin Cre lines (43) has been reported to result in both hematopoietic and vascular defects, including anaemia, hemorrhage, edema, and blood-filled lymphatic vessels. Targeted deletion of the Gata $2+9.5 \mathrm{~kb}$ regulatory element, essentially ablating Gata2 in the endothelial and hematopoietic lineages, results in similar phenotypes (14). Our documentation of aberrant LVV formation in Gata2 ${ }^{\triangle E C}$ mice likely explains the phenotypes of edema and blood-filled lymphatic vessels observed in the mutant mice reported in earlier studies, though we can't at present discount a potential contribution from hematopoietic cell defects to this phenotype. On the basis of published work, the lineage most likely to contribute to blood-filled lymphatics is the megakaryocyte/platelet lineage, recently documented to function together with the LVV to prevent the entry of blood cells to the lymphatic vasculature (35), and a lineage in which Gata2 has documented roles $(45,58)$.

Intriguingly, blood-filled lymph sacs and lymphatic vessels were prominent in E13.5 Gata ${ }^{4 L E C}$ embryos in which Gata2 excision was induced by the administration of tamoxifen at E10.5, E11.5, and E12.5, but were not obvious in E14.5 Gata ${ }^{\triangle L E C}$ embryos in which Gata2 excision was induced at E10.5 and E11.5. Accordingly, no prominent defects in LVV formation were observed in these E14.5 Gata ${ }^{\triangle L E C}$ mice. Though disruption to tissue morphology in E13.5 Gata $^{4 L E C}$ embryos has restricted rigorous analysis of LVV morphology, we expect that either the structure or function of this valve is impaired, resulting in the blood-filled lymphatic phenotype. The presence of edema in Gata ${ }^{\triangle L E C}$ embryos at both E13.5 and E14.5, even in the absence of blood-filled jugular lymph sacs, is suggestive of lymphatic vessel dysfunction and a potential role for Gata 2 in the lymphatic vasculature prior to the initiation of lymphatic vessel valve development. Our analyses of Gata2 exci- 


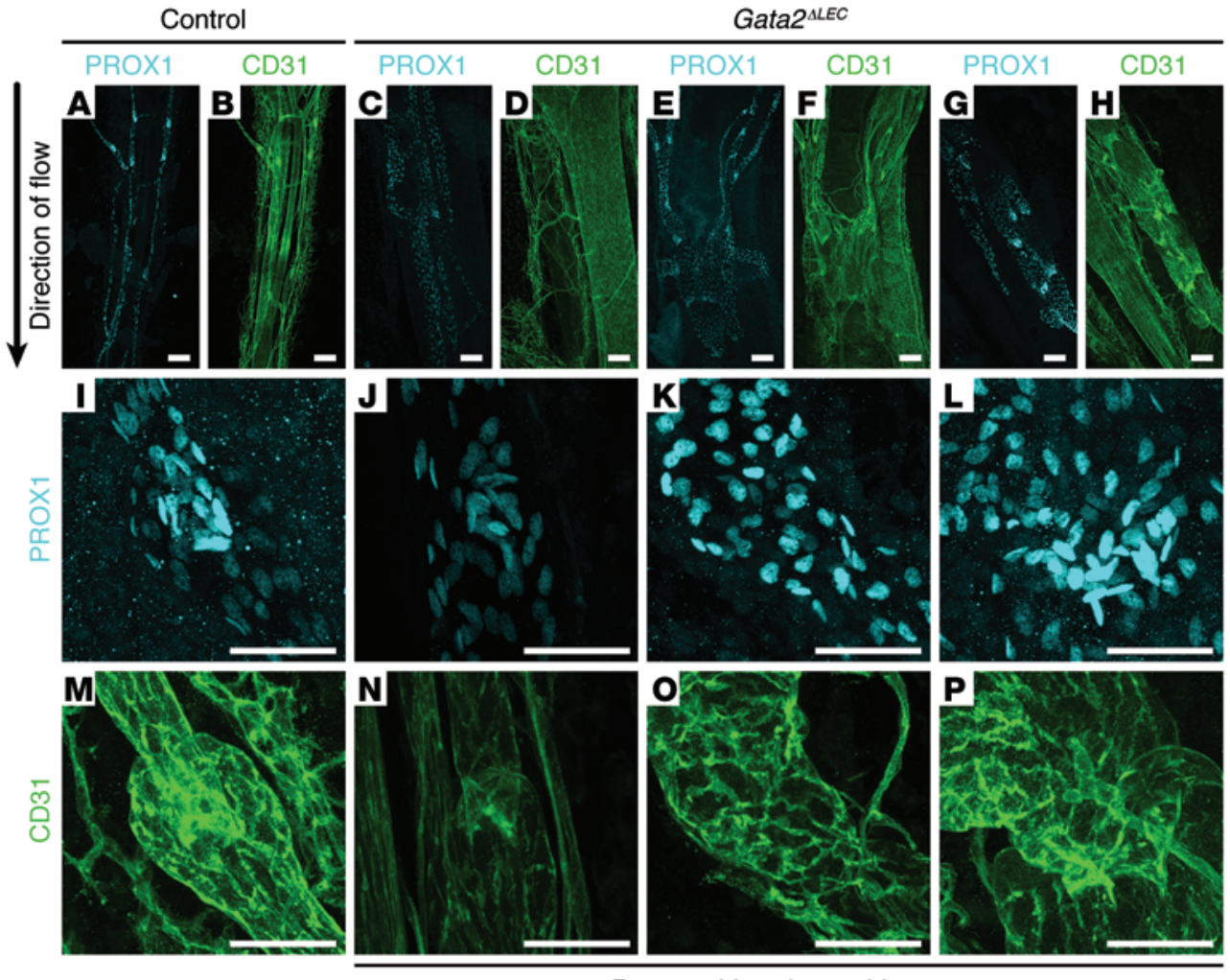

Dysmorphic valve architecture
Figure 11. Gata2 deletion results in degeneration of lymphatic vessel valves and lymphatic vessel distension. Gata ${ }^{\triangle L E C}$ and littermate control (Cre-negative; Gata $2^{f / f f}$ ) pups were injected with tamoxifen at P4. Wholemount immunostaining of mesenteries at P10 with PROX1 (cyan) and CD31 (green) demonstrated severely dysmorphic lymphatic vessel valves and distended lymphatic vessels in Cata2 $2^{\triangle L E C}$ mesenteries (C-H, J-L, and $\mathbf{N}-\mathbf{P}$ ), compared with littermate controls (A, B, I, and $\mathbf{M})$. Scale bars: $100 \mu \mathrm{m}(\mathbf{A}-\mathbf{H})$ or $50 \mu \mathrm{m}(\mathbf{I}-\mathbf{P})$ sion in the LVV region of E13.5 and E14.5 Gata ${ }^{4 L E C}$ embryos suggests that tamoxifen administration at E12.5 might be critical for efficient Gata2 deletion in the LVV using the Prox1-Cre ${ }^{E R T 2}$ line. In support of this possibility, our analysis of $\beta$-galactosidase levels in the LVV region of E14.5 Prox1-Cre ${ }^{E R T 2}$ ROSA26R embryos following one tamoxifen administration at E12.5 revealed that the majority of cells in the LVV region were labeled.

While a number of recent studies have contributed to our understanding of how PROX1 transcription is controlled, there remains a dearth of knowledge in this arena. Our data identify GATA2 as an important transcriptional regulator of PROX1 and reveal a novel enhancer element $11 \mathrm{~kb}$ upstream of the first, noncoding exon of PROX1 that is bound by GATA2, FOXC2, and NFATC1. Given our data demonstrating that Prox1 expression is still initiated in lymphatic endothelial progenitor cells in the cardinal veins in the absence of Gata2, we hypothesize that the binding of Gata 2 to the $-11 \mathrm{~kb}$ enhancer element is not required to "switch on" Prox1, but rather is important for "dialling up" Prox1 levels in valve-forming cells. There seems little doubt that transcriptional cofactors, in addition to GATA2, are required to coordinate $P R O X 1$ transcription differentially in LECs and BECs, since we detected binding of GATA2 at the PROX1-11 kb locus in both cell types, though at a greater magnitude in hLECs. In support of this hypothesis, ChIP studies identified differences in the chromatin architecture of the PROX1-11 kb locus in hLECs compared with hBECs. Monomethylation of H3K4Me1, a mark indicative of active or poised enhancer elements (59), was associated with the PROX1-11 kb locus in hLECs and, to a lesser extent, in hBEC; however, it was not present in K562 cells, an erythroid cell line negative for PROX1. In contrast, trimethylation of $\mathrm{H} 3 \mathrm{~K} 27 \mathrm{Me} 3-\mathrm{a}$ marker of repressed, inactive chromatin (60) - was not detected at PROX1-11 kb in hLECs but was prominent in both hBECs and K562 cells. Taken together, these data suggest that GATA2 may be poised at the PROX1 $-11 \mathrm{~kb}$ enhancer in BECs and that yetto-be-identified chromatin remodeling/transcription factors are important for switching this enhancer to the "on" state in LECs. An important answer to the question of how PROX1 transcription is temporally and spatially controlled will come from defining the relative contribution of the $-11 \mathrm{~kb}$ element compared with other potential enhancer elements.

How is it that GATA2 levels are distinctly higher in valveforming territories? Our data suggest that at least one mechanism responsible for the elevation of GATA2 levels in lymphatic vessel and LVV valves is mechanical in nature and mediated by OSS, though additional stimuli are likely involved in regulating GATA2 levels within the lymphatic vasculature and between distinct vascular endothelial compartments. Established regulators of GATA2 transcription include GATA2 itself, as well as GATA1, reported to repress GATA2 expression in hematopoietic cells (37). GATA2 is both positively and negatively regulated by the Notch signalling pathway; NOTCH1/RBJк is required to initiate Gata2 expression in hematopoietic stem cells in the embryonic aorta-gonadmesonephros region (61), while the Notch-induced gene Hes1 subsequently negatively regulates Gata2 in hematopoietic stem cells of the AGM, controlling the production of functional HSC (62). NOTCH1 function has recently been shown to be important for lymphatic vessel valve development; loss of NOTCH1 results in fewer valves, disrupted reorientation of valve endothelial cells, and reduced levels of valve markers, including ITG $\alpha 9$ and FN-EIIIA (25). Whether or not Notch signalling is important 


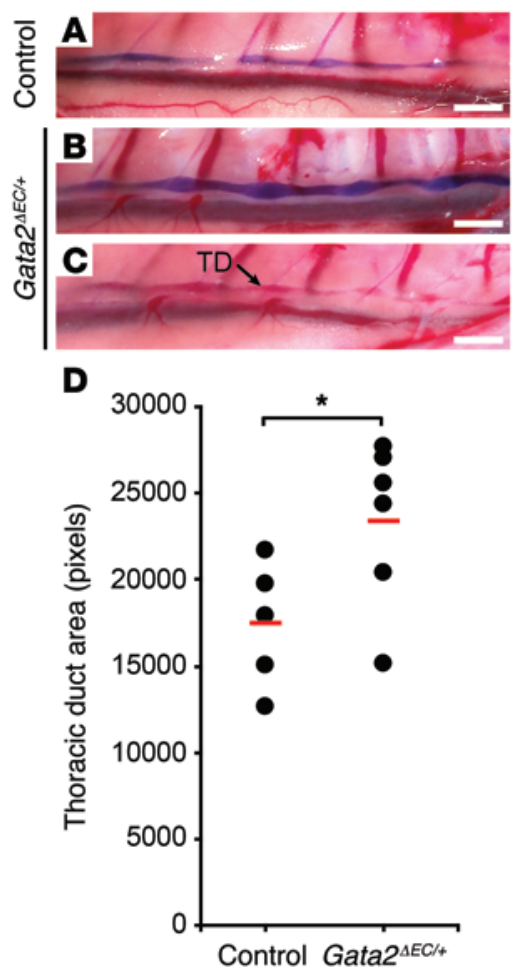

for the control of Gata2 levels in valve endothelial cells remains to be assessed. Other signalling axes that regulate Gata2 expression include BMP signalling, required to induce Gata2 and specify ventral identity in Xenopus (63), and retinoic acid (RA) signalling, shown to impact on the transcriptional activity of GATA2 by virtue of a direct interaction between the zinc fingers of GATA2 and the DNA-binding domain of RA receptor $\alpha(\operatorname{RAR} \alpha)$ (64). Of interest, BMP9 has recently been shown to control lymphatic vessel valve formation (31), and our own recent work revealed aberrant development of LVVs in Cyp26b1/-/ mice in which RA signalling is elevated (65). Potential regulation of GATA2 by both of these pathways will be the subject of future investigation.

Recent large-scale ChIP-Seq studies analyzing the binding of multiple transcription factors across distinct hematopoietic cell types have revealed key insights to the identity of transcriptionfactor complexes and target genes important for programming cell identity $(44,45)$. Such studies in hematopoietic cell lineages have revealed that GATA2 works cooperatively with transcription factors, including RUNX1, SCL/TAL1, FLI1, LYL1, LMO2, and ERG $(44,45)$. The identity of GATA2 transcriptional cofactors in endothelial cells and, in particular, in lymphatic versus blood vas-

Figure 13. Model depicting mechanisms by which Gata2 regulates lymphatic vascular development. GATA2 is elevated in valve-forming territories at the onset of lymphatic vessel valve development and regulates the levels of PROX1 and FOXC2 in valve-forming endothelial cells. Deletion of Gata2 in the lymphatic vasculature prior to the initiation of lymphatic vessel valve development results in reduced FOXC2 levels throughout the lymphatic vasculature, ectopic recruitment of vascular SMCs to dermal lymphatic vessels, dilated lymphatic vessels, and arrested valve development. Postnatal deletion of Gata2 in the lymphatic vasculature following valve assembly results in diminished levels of PROX1 in the endothelial cells comprising lymphatic vessel valves and consequent valve disorganization.
Figure 12. Lymphatic vascular defects in adult Gata2 ${ }^{4 E C}$ mice. Adult heterozygous Gata2 ${ }^{4 E C /+}$ mice injected with Evans Blue dye exhibited collecting lymphatic vessels of substantially larger caliber (B-D) than controls (A). Thoracic duct area was measured using Imagej in control $(n=5)$ and heterozygous Cata $2^{\operatorname{LEC} /+}(n=6)$ adult mice $(\mathbf{D}) .{ }^{*} P<0.05$, by 2 -tailed Student's $t$-test. Reduced transport of Evans Blue dye to the thoracic duct and blood within the thoracic duct (C; arrow) were also observed in Gata $2^{4 E C /+}$ mice. Scale bars: $1 \mathrm{~mm}$. TD, thoracic duct.

cular endothelial cells remains to be established and is an avenue of research we are actively pursuing. At the posttranslational level, GATA2 is reportedly subject to control by phosphorylation, acetylation, and sumoylation (37), though whether any of these modifications has an impact on the high levels of nuclear GATA2 present in valve-forming territories remains to be determined.

Our assessment of the impact of germline GATA2 mutations found in Emberger syndrome on protein structure, compared with those found only in hematological disorders (germline or somatic), has provided insight to the reasons why some but not all germline GATA2 mutations result in lymphedema. Analysis of the structure of the GATA2 zinc fingers containing Emberger mutations R361L, C373R, and R396Q, together with their binding to the GATA site in the PROX1 -11 kb enhancer element, revealed that these mutations exhibit a near complete ablation of DNA binding due to alteration in key DNA-binding residues or severe disruption to protein folding. In contrast, GATA2 mutants associated with hematological disorders but not lymphedema retained some capacity to bind the PROX1-11 kb enhancer. This finding is in agreement with hypotheses proposed by us (3) and others (49), suggesting that effectively null haploinsufficiency of GATA2 is the critical factor predisposing to lymphedema onset. We don't rule out the possibility, however, that additional insults such as infection, defective immune cell trafficking resulting in inflammation, and/or injury may contribute to lymphedema onset in patients. It remains enigmatic that mutations such as $355 \mathrm{del}$, essentially loss of function in all assays tested, are not to date associated with lymphedema. Additional phenotypes reported in patients carrying GATA2 mutations/deletions that may reflect a role for GATA2 as a general regulator of valve development and function include venous thromboses and culture-negative

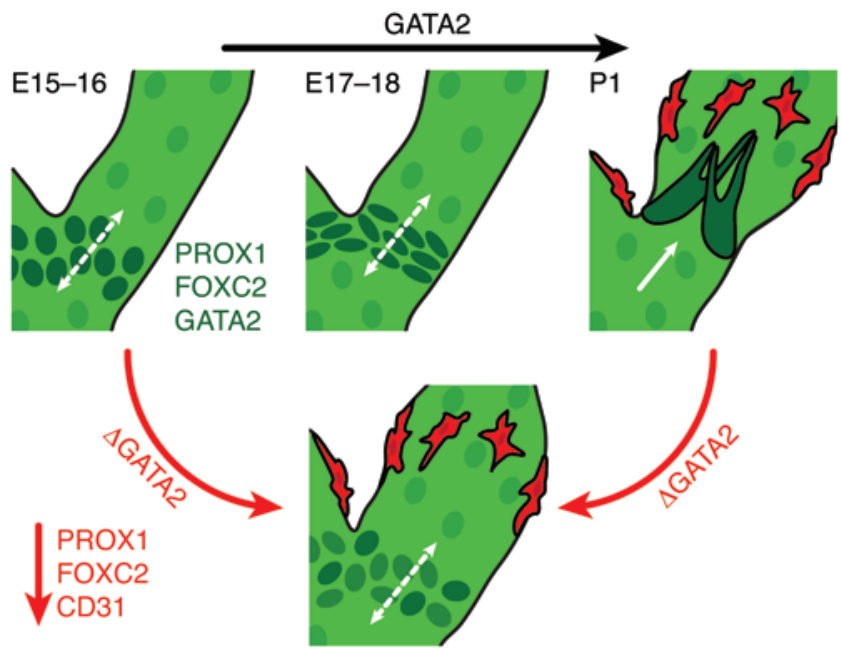


endocarditis (49). Since many of the genes and cellular events important for lymphatic vessel valve development are also important for venous valve development (32), it stands to reason that venous valve development and/or function may also be affected in patients with GATA2 mutations, a scenario that may contribute to venous thromboses. Likewise, our demonstration that Gata2 is also present in cardiac valves suggests that GATA2 mutations may potentially result in defective cardiac valve development, one of the factors underlying culture-negative endocarditis. An important role for a second GATA family member, Gata4, has been documented during atrioventricular valve development in mice (66) and mutations in GATA 4 have been associated with human atrial and ventricular septal defects and pulmonary valve stenosis (67).

In conclusion, our study defines a crucial role for Gata 2 in lymphatic vascular development and reveals that Gata2 is essential for the morphogenesis of lymphovenous and lymphatic vessel valves. We provide new insight to the mechanisms by which selected GATA2 mutations result in lymphedema in human patients, demonstrating that complete loss of transcriptional activity with respect to the control of genes important for lymphatic vessel valve development underlies this phenotype. Our work has identified a potentially "valve selective" enhancer element $11 \mathrm{~kb}$ upstream of the PROX1 locus that is bound by GATA2, FOXC2, and NFATC1 in endothelial cells and that is, in general, "on" in lymphatic endothelial cells and "poised" in blood vascular endothelial cells, at the epigenetic level. Our work sets the stage for future investigations to define the precise mechanisms, in terms of the transcriptional cofactors and target genes of GATA2, that are important for the process of valve development and has the potential to uncover new targets to which the design of novel agents able to promote valve development and/or function could be designed.

\section{Methods}

For a full description of Methods, see Supplemental Methods online. Mice. Prox1-Cre ${ }^{E R T 2}$ (56) Gata2 ${ }^{f / f l}$ (55), Tie2-Cre (54), and ROSA26R Cre reporter (68) mice have been previously described.
ChIP-Seq data accession. ChIP-Seq data has been deposited in the European Nucleotide Archive (ENA), accession number PRJEB9436 (http://www.ebi.ac.uk/ena/data/view/PRJEB9436).

Statistics. Analysis of luciferase activity of all GATA2 mutants compared with WT (shown in Figure 1E and Supplemental Figure 1B) was performed using 1-way ANOVA with Dunnett's multiple-comparisons test. For all other statistical analyses, $P$ values were calculated using the 2-tailed Student's $t$ test. $P$ values of less than 0.05 were considered statistically significant.

Study approval. Experiments using mice were approved and conducted in accordance with the SA Pathology/CALHN Animal Ethics Committee and Australian National Health and Medical Research Council (NHMRC) guidelines.

\section{Acknowledgments}

We thank Sylvia Tichborne, Chris Brown, Kelly Wicks, and staff at the SA Pathology Animal Facility for animal husbandry; Guillermo Oliver for Prox1-Cre ${ }^{E R T 2}$ mice; Leigh Coultas for Tie2-Cre mice; Mat Francois for the pGL2BasicProx1-4kbintron1 construct; Junia Melo for K562 cells; Stuart Pitson for HEK293 cells; and John Pimanda, Guillermo Oliver, and Brian Black for helpful discussions. This work was supported by project grants from the NHMRC (APP1061365, to N.L. Harvey and H.S. Scott; APP1004651, to J.M. Matthews), South Australian Health \& Medical Research Institute Beat Cancer Project (to N.L. Harvey and H.S. Scott), and Swiss National Science Foundation (CRII3 141811, to T.V. Petrova). N.L. Harvey is supported by an ARC Future Fellowship. J.M. Matthews is a Senior Research Fellow and HSS, a Principal Research Fellow of the NHMRC. C.S. Demir was supported by the People Programme (Marie Curie Actions) of the European Union's Seventh Framework Programme FP7 (2007-2013) under REA grant agreement 317250.

Address correspondence to: Natasha Harvey, Centre for Cancer Biology, UniSA and SA Pathology, PO Box 14, Rundle Mall, Adelaide, South Australia, 5000, Australia. Phone: 61.8.8222.3569; E-mail: Natasha.harvey@unisa.edu.au.
1. Mansour S, et al. Emberger syndromeprimary lymphedema with myelodysplasia: report of seven new cases. Am JMed Genet A. 2010;152A(9):2287-2296.

2. Ostergaard P, et al. Mutations in GATA2 cause primary lymphedema associated with a predisposition to acute myeloid leukemia (Emberger syndrome). Nat Genet. 2011;43(10):929-931.

3. Kazenwadel J, et al. Loss-of-function germline GATA2 mutations in patients with MDS/AML or MonoMAC syndrome and primary lymphedema reveal a key role for GATA2 in the lymphatic vasculature. Blood. 2012;119(5):1283-1291.

4. Ishida $H$, et al. GATA-2 anomaly and clinical phenotype of a sporadic case of lymphedema, dendritic cell, monocyte, B- and NK-cell (DCML) deficiency, and myelodysplasia. Eur JPediatr. 2012;171(8):1273-1276

5. Khandekar M, et al. A Gata2 intronic enhancer confers its pan-endothelia-specific regulation. Development. 2007;134(9):1703-1712.

6. Mammoto A, et al. A mechanosensitive transcriptional mechanism that controls angiogenesis.
Nature. 2009;457(7233):1103-1108.

7. Pimanda JE, et al. Endoglin expression in blood and endothelium is differentially regulated by modular assembly of the Ets/Gata hemangioblast code. Blood. 2008;112(12):4512-4522.

8. Linnemann AK, O'Geen H, Keles S, Farnham PJ, Bresnick EH. Genetic framework for GATA factor function in vascular biology. Proc Natl Acad Sci US A. 2011;108(33):13641-13646.

9. Muiya NP, et al. A study of the role of GATA2 gene polymorphism in coronary artery disease risk traits. Gene. 2014;544(2):152-158.

10. Connelly JJ, et al. GATA2 is associated with familial early-onset coronary artery disease. PLOS Genet. 2006;2(8):e139.

11. Liu YH, et al. Gene polymorphisms associated with susceptibility to coronary artery disease in Han Chinese people. Genet Mol Res. 2014;13(2):2619-2627.

12. Zhu C, et al. Evaluation and application of modularly assembled zinc-finger nucleases in zebrafish. Development. 2011;138(20):4555-4564.

13. Lim KC, et al. Conditional Gata2 inactivation results in HSC loss and lymphatic mispatterning J Clin Invest. 2012;122(10):3705-3717.

14. Johnson KD, et al. Cis-element mutated in GATA2-dependent immunodeficiency governs hematopoiesis and vascular integrity. J Clin Invest. 2012;122(10):3692-3704.

15. Petrova TV, et al. Defective valves and abnormal mural cell recruitment underlie lymphatic vascular failure in lymphedema distichiasis. Nat Med. 2004;10(9):974-981.

16. Sabine A, Petrova TV. Interplay of mechanotransduction, FOXC2, connexins, and calcineurin signaling in lymphatic valve formation. Adv Anat Embryol Cell Biol. 2014;214:67-80.

17. Bazigou E, Makinen T. Flow control in our vessels: vascular valves make sure there is no way back. Cell Mol Life Sci. 2012;70(6):1055-1066.

18. Koltowska K, Betterman KL, Harvey NL, Hogan BM. Getting out and about: the emergence and morphogenesis of the vertebrate lymphatic vasculature. Development. 2013;140(9):1857-1870.

19. Bazigou E, et al. Integrin-alpha9 is required for fibronectin matrix assembly during lymphatic valve 
morphogenesis. Dev Cell. 2009;17(2):175-186.

20. Sabine A, et al. Mechanotransduction, PROX1, and FOXC2 cooperate to control connexin 37 and calcineurin during lymphatic-valve formation. Dev Cell. 2012;22(2):430-445.

21. Danussi C, et al. EMILIN1/alpha9beta1 integrin interaction is crucial in lymphatic valve formation and maintenance. Mol Cell Biol. 2013;33(22):4381-4394.

22. Norrmen C, et al. FOXC2 controls formation and maturation of lymphatic collecting vessels through cooperation with NFATc1.J Cell Biol. 2009;185(3):439-457.

23. Makinen T, et al. PDZ interaction site in ephrinB2 is required for the remodeling of lymphatic vasculature. Genes Dev. 2005;19(3):397-410.

24. Kanki Y, et al. Epigenetically coordinated GATA2 binding is necessary for endothelium-specific endomucin expression. $E M B O J$. 2011;30(13):2582-2595.

25. Murtomaki A, et al. Notch signaling functions in lymphatic valve formation. Development. 2014;141(12):2446-2451.

26. Jurisic $\mathrm{G}$, et al. An unexpected role of semaphorin3a-neuropilin-1 signaling in lymphatic vessel maturation and valve formation. Circ Res. 2012;111(4):426-436.

27. Bouvree K, et al. Semaphorin3A, Neuropilin-1, and PlexinA1 are required for lymphatic valve formation. Circ Res. 2012;111(4):437-445.

28. Gale NW, et al. Angiopoietin-2 is required for postnatal angiogenesis and lymphatic patterning, and only the latter role is rescued by Angiopoietin-1. Dev Cell. 2002;3(3):411-423.

29. Dellinger $M$, et al. Defective remodeling and maturation of the lymphatic vasculature in Angiopoietin-2 deficient mice. Dev Biol. 2008;319(2):309-320.

30. Shen B, et al. Genetic dissection of tie pathway in mouse lymphatic maturation and valve development. Arterioscler Thromb Vasc Biol. 2014;34(6):1221-1230.

31. Levet $\mathrm{S}$, et al. Bone morphogenetic protein 9 (BMP9) controls lymphatic vessel maturation and valve formation. Blood. 2013;122(4):598-607.

32. Bazigou E, et al. Genes regulating lymphangiogenesis control venous valve formation and maintenance in mice. J Clin Invest. 2011;121(8):2984-2992.

33. Srinivasan RS, Oliver G. Prox1 dosage controls the number of lymphatic endothelial cell progenitors and the formation of the lymphovenous valves. Genes Dev. 2011;25(20):2187-2197.

34. van der Putte SC. The early development of the lymphatic system in mouse embryos. Acta Morphol Neerl Scand.1975;13(4):245-286.

35. Hess PR, et al. Platelets mediate lymphovenous hemostasis to maintain blood-lymphatic separation throughout life. J Clin Invest. 2014;124(1):273-284.

36. Turner CJ, Badu-Nkansah K, Crowley D, van der
Flier A, Hynes RO. Integrin- $\alpha 5 \beta 1$ is not required for mural cell functions during development of blood vessels but is required for lymphatic-blood vessel separation lymphovenous valve formation. Dev Biol. 2014;392(2):381-392.

37. Bresnick EH, Katsumura KR, Lee HY, Johnson KD, Perkins AS. Master regulatory GATA transcription factors: mechanistic principles and emerging links to hematologic malignancies. Nucleic Acids Res. 2012;40(13):5819-5831.

38. Charles MA, et al. Pituitary-specific Gata2 knockout: effects on gonadotrope and thyrotrope function. Mol Endocrinol. 2006;20(6):1366-1377.

39. Kala K, et al. Gata2 is a tissue-specific postmitotic selector gene for midbrain GABAergic neurons. Development. 2009;136(2):253-262.

40. Zhou Y, et al. Rescue of the embryonic lethal hematopoietic defect reveals a critical role for GATA-2 in urogenital development. EMBO J. 1998;17(22):6689-6700.

41. Tsai FY, et al. An early haematopoietic defect in mice lacking the transcription factor GATA-2. Nature. 1994;371(6494):221-226.

42. Rodrigues NP, et al. Haploinsufficiency of GATA-2 perturbs adult hematopoietic stem-cell homeostasis. Blood. 2005;106(2):477-484.

43. de Pater E, et al. Gata2 is required for HSC generation and survival. J Exp Med. 2013;210(13):2843-2850.

44. Wilson NK, et al. Combinatorial transcriptional control in blood stem/progenitor cells: genomewide analysis of ten major transcriptional regulators. Cell Stem Cell. 2010;7(4):532-544.

45. Tijssen MR, et al. Genome-wide analysis of simultaneous GATA1/2, RUNX1, FLI1, and SCL binding in megakaryocytes identifies hematopoietic regulators. Dev Cell. 2011;20(5):597-609.

46. Fujiwara T, et al. Discovering hematopoietic mechanisms through genome-wide analysis of GATA factor chromatin occupancy. Mol Cell. 2009;36(4):667-681.

47. Martin DI, Orkin SH. Transcriptional activation and DNA binding by the erythroid factor GF-1/ NF-E1/Eryf 1. Genes Dev. 1990;4(11):1886-1898.

48. Omichinski JG, et al. NMR structure of a specific DNA complex of Zn-containing DNA binding domain of GATA-1. Science. 1993;261(5120):438-446.

49. Spinner MA, et al. GATA2 deficiency: a protean disorder of hematopoiesis, lymphatics, and immunity. Blood. 2013;123(6):809-821.

50. Francois M, et al. Sox18 induces development of the lymphatic vasculature in mice. Nature. 2008;456(7222):643-647.

51. Hahn CN, et al. Heritable GATA2 mutations associated with familial myelodysplastic syndrome and acute myeloid leukemia. Nat Genet. 2011;43(10):1012-1017.

52. Chen Y, et al. DNA binding by GATA transcription factor suggests mechanisms of DNA looping and long-range gene regulation. Cell Rep.
2012;2(5):1197-1206.

53. Wigle JT, Oliver G. Prox1 function is required for the development of the murine lymphatic system. Cell. 1999;98(6):769-778.

54. Kisanuki YY, Hammer RE, Miyazaki J, Williams SC, Richardson JA, Yanagisawa M. Tie2-Cre transgenic mice: a new model for endothelial cell-lineage analysis in vivo. Dev Biol. 2001;230(2):230-242.

55. Haugas M, Lillevali K, Hakanen J, Salminen M. Gata2 is required for the development of inner ear semicircular ducts and the surrounding perilymphatic space. Dev Dyn. 2010;239(9):2452-2469.

56. Srinivasan RS, et al. Lineage tracing demonstrates the venous origin of the mammalian lymphatic vasculature. Genes Dev. 2007;21(19):2422-2432.

57. Grass JA, et al. Distinct functions of dispersed GATA factor complexes at an endogenous gene locus. Mol Cell Biol. 2006;26(19):7056-7067.

58. Huang Z, et al. GATA-2 reinforces megakaryocyte development in the absence of GATA-1. Mol Cell Biol. 2009;29(18):5168-5180.

59. Heintzman ND, et al. Distinct and predictive chromatin signatures of transcriptional promoters and enhancers in the human genome. Nat Genet. 2007;39(3):311-318.

60. Cao R, et al. Role of histone $\mathrm{H} 3$ lysine 27 methylation in Polycomb-group silencing. Science. 2002;298(5595):1039-1043.

61. Robert-Moreno A, Espinosa L, de la Pompa JL, Bigas A. RBPjkappa-dependent Notch function regulates Gata2 and is essential for the formation of intra-embryonic hematopoietic cells. Development. 2005;132(5):1117-1126.

62. Guiu J, et al. Hes repressors are essential regulators of hematopoietic stem cell development downstream of Notch signaling. J Exp Med. 2013;210(1):71-84.

63. Oren T, Torregroza I, Evans T. An Oct-1 binding site mediates activation of the gata2 promoter by BMP signaling. Nucleic Acids Res. 2005;33(13):4357-4367.

64. Tsuzuki S, Kitajima K, Nakano T, Glasow A, Zelent A, Enver T. Cross talk between retinoic acid signaling and transcription factor GATA-2. Mol Cell Biol. 2004;24(15):6824-6836.

65. Bowles J, et al. Control of retinoid levels by CYP26B1 is important for lymphatic vascular development in the mouse embryo. Dev Biol. 2013;386(1):25-33.

66. Rivera-Feliciano J, et al. Development of heart valves requires Gata 4 expression in endothelial-derived cells. Development. 2006;133(18):3607-3618.

67. Garg V, et al. GATA4 mutations cause human congenital heart defects and reveal an interaction with TBX5. Nature. 2003;424(6947):443-447.

68. Soriano P. Generalized lacZ expression with the ROSA26 Cre reporter strain. Nat Genet. 1999;21(1):70-71. 